\title{
THE ALHAMBRA SURVEY: A LARGE AREA MULTIMEDIUM-BAND OPTICAL AND NEAR-INFRARED PHOTOMETRIC SURVEY*
}

\author{
M. Moles ${ }^{1}$, N. Benítez ${ }^{1,2}$, J. A. L. Aguerri ${ }^{3}$, E. J. Alfaro ${ }^{1}$, T. Broadhurst ${ }^{4}$, J. Cabrera-Caño ${ }^{5}$, F. J. Castander ${ }^{6}$, \\ J. Cepa ${ }^{3,7}$, M. Cerviño ${ }^{1}$, D. Cristóbal-Hornillos ${ }^{1}$, A. Fernández-Soto ${ }^{8}$, R. M. GonZález Delgado ${ }^{1}$, L. Infante ${ }^{9}$, \\ I. Márquez ${ }^{1}$, V. J. Martínez ${ }^{8,10}$, J. Masegosa ${ }^{1}$, A. Del Olmo ${ }^{1}$, J. Perea ${ }^{1}$, F. Prada ${ }^{1}$, J. M. Quintana ${ }^{1}$, and S. F. Sánchez ${ }^{11}$ \\ ${ }^{1}$ Instituto de Astrofísica de Andalucía, CSIC, Apdo. 3044, E-18080 Granada; moles@iaa.es, emilio@iaa.es, mcs@iaa.es, dch@iaa.es, rosa@iaa.es, isabel@iaa.es, \\ pepa@iaa.es, chony@iaa.es, jaime@iaa.es, fprada@iaa.es, and quintana@iaa.es \\ ${ }^{2}$ Instituto de Matemáticas y Física Fundamental, CSIC, Serrano 113-bis, Madrid 28006, Spain; benitez@iaa.es \\ ${ }^{3}$ Instituto de Astrofísica de Canarias, La Laguna, Spain; jalfonso@iac.es \\ ${ }^{4}$ School of Physics and Astronomy, Tel Aviv University, Israel; tjb @ wise1.tau.ac.il \\ ${ }^{5}$ Departamento de Física Atómica, Molecular y Nuclear, Facultad de Física, Universidad de Sevilla, Spain; jcc-famn@us.es \\ ${ }^{6}$ Institut de Ciències de l’Espai, IEEC-CSIC, Barcelona, Spain; fjc@ieec.fcr.es \\ ${ }^{7}$ Departamento de Astrofísica, Facultad de Física, Universidad de la Laguna, Spain; jen@iac.es \\ ${ }^{8}$ Departament d'Astronomía i Astrofísica, Universitat de València, València, Spain; alberto.fernandez@uv.es \\ ${ }_{9}^{9}$ Departamento de Astronomía, Pontificia Universidad Católica, Santiago, Chile; linfante@ astro.puc.cl \\ ${ }^{10}$ Observatori Astronòmic de la Universitat de València, València, Spain; vicent.martinez@uv.es \\ ${ }^{11}$ Centro Astronómico Hispano-Alemán, Almería, Spain; sanchez@caha.es \\ Received 2007 February 7; accepted 2008 June 17; published 2008 August 15
}

\begin{abstract}
Here we describe the first results of the Advanced Large Homogeneous Area Medium-Band Redshift Astronomical (ALHAMBRA) survey, which provides cosmic tomography of the evolution of the contents of the universe over most of cosmic history. Our novel approach employs 20 contiguous, equal-width, medium-band filters covering from $3500 \AA$ to $9700 \AA$, plus the standard $J H K_{s}$ near-infrared (NIR) bands, to observe a total area of $4 \mathrm{deg}^{2}$ on the sky. The optical photometric system has been designed to maximize the number of objects with accurate classification by spectral energy distribution type and redshift, and to be sensitive to relatively faint emission features in the spectrum. The observations are being carried out with the Calar Alto $3.5 \mathrm{~m}$ telescope using the wide-field cameras in the optical, Large Area Imager for Calar Alto, and in the NIR, Omega-2000. The first data confirm that we are reaching the expected magnitude limits (for a total of $100 \mathrm{ks}$ integration time per pointing) of $A B \leqslant 25 \mathrm{mag}$ (for an unresolved object, signal-to-noise ratio $=5$ ) in the optical filters from the blue to $8300 \AA$, and from $A B=24.7$ to 23.4 for the redder ones. The limit in the NIR, for a total of $15 \mathrm{ks}$ exposure time per pointing, is (in the Vega system) $K_{s} \approx 20 \mathrm{mag}$, $H \approx 21 \mathrm{mag}, J \approx 22 \mathrm{mag}$. Some preliminary results are presented here to illustrate the capabilities of the ongoing survey. We expect to obtain accurate redshift values, $\Delta z /(1+z) \leqslant 0.03$ for about five $\times 10^{5}$ galaxies with $I \leqslant 25(60 \%$ completeness level), and $z_{\text {med }}=0.74$. This accuracy, together with the homogeneity of the selection function, will allow for the study of the redshift evolution of the large-scale structure, the galaxy population and its evolution with redshift, the identification of clusters of galaxies, and many other studies, without the need for any further follow-up. It will also provide targets for detailed studies with $10 \mathrm{~m}$ class telescopes. Given its area, spectral coverage, and its depth, apart from those main goals, the ALHAMBRA survey will also produce valuable data for galactic studies.
\end{abstract}

Key words: cosmology: observations - galaxies: evolution - surveys - techniques: photometric

Online-only material: color figures

\section{INTRODUCTION: GLOBAL SCIENTIFIC AIM AND OPPORTUNITY}

Only over the last few years has it become possible for Observational Cosmology to gather enough data on the distant universe to feed our comprehension of the evolution of the different objects that populate it. It has become almost commonplace to study protogalaxies at redshifts $z>5$, and to observe particular objects at redshifts as high as $z \approx 6.5$ (Becker et al. 2001; Kashikawa et al. 2006; Kawai et al. 2006) or even $z \approx 7.5$ (Bradley et al. 2008). At the same time, samples of objects have been collected through different techniques at smaller distances (and shorter evolutionary times) from us, and the different properties of objects in separate redshift ranges have been measured and compared. However, it remains true that to this day, no

\footnotetext{
* Based on observations collected at the German-Spanish Astronomical Center, Calar Alto, jointly operated by the Max-Planck-Institut für Astronomie Heidelberg and the Instituto de Astrofísica de Andalucía (CSIC).
}

homogeneous sample of objects has been collected covering a significant range of the age of the universe, even if some remarkable efforts have been devoted to the production of widefield, shallow surveys, that cover the low-redshift end (such as 2dF Galaxy Redshift Survey (GRS), Colless et al. 2001; Sloan Digital Sky Survey (SDSS), York et al. 2000; VIRMOS-VLT Deep Survey (VVDS), Le Fèvre et al. 2005; or Deep Extragalactic Evolutionary Probe 2 (DEEP 2) Redshift Survey, Davis et al. 2003) while other groups have directed their efforts toward the most distant end, through very deep, small-area surveys such as the Hubble Space Telescope (HST) Deep Fields or other legacy programs (Ferguson et al. 2000; Beckwith et al. 2006).

The cosmological principle implies the existence of maximally symmetric subspaces and the existence of a one-to-one relation between redshift and time. The corresponding evolutionary nature of the depicted universe is a model-independent prediction, prior to any consideration about the value of the cosmological parameters. Hence, a direct way to tackle many 
of the problems posed by modern cosmology is to materialize a foliation of the space-time, producing narrow slices in the $z$-direction whereas the spatial sections are large enough to be cosmologically representative, obtaining as output a kind of cosmic tomography.

From the observational point of view, to trace cosmic evolution, which is a central topic in cosmology, the genuine evolutionary effects have to be disentangled from both the physical variance at a given redshift and the details of the metric as measured in —or, depending on the point of view, induced by - the cosmological model. In other words, to approach the question of evolution meaningfully, it is necessary to sample large volumes even at low redshift, to capture not only representative average properties but also their variance. This will necessarily imply a survey featuring a combination of wide area and depth, and a continuous spectral coverage to avoid complex selection functions that depend on the redshift and on the nature of the objects under analysis. Moreover, the quest for the necessary precision implies high enough spectral resolution and photometric accuracy.

Up to now, the largest surveys ensuring complete spectral coverage for large samples have been photometric, and done with broadband filters. The resulting redshift precision obtained with these techniques $(\sim 0.03$ in $\Delta z /(1+z)$ at best; see Cucciati et al. 2006; Ilbert et al. 2006) and in spectral energy distribution (SED) determination are correspondingly rough. Moreover, large area photometric surveys such as the Sloan Digital Sky Survey (SDSS) are necessarily shallow, whereas deeper surveys have sampled the distant and/or faint universe in rather small areas. At the other extreme in spectral resolution, spectroscopic surveys can neither go as deep as the photometric ones nor cover large enough areas. Moreover, they are defined in order to observe a restricted spectral region, producing a selection effect that is a function of the object type and redshift that can be very intricate due to the selection effects inherent to spectroscopy (Fernández-Soto et al. 2001).

For those scientific purposes where the detailed properties of individual objects are not the goal, the aim from an observational standpoint is therefore that of finding the optimal filter combination to produce the most homogeneous, deepest, and most accurate possible photometric survey. Such a survey would produce precise enough values for the redshift and SED for large numbers of objects. We present here the Advanced Large Homogeneous Area Medium-Band Redshift Astronomical (ALHAMBRA) survey, that intends to produce such an optimum survey for the study of cosmic evolution. It has been designed to achieve (with the facilities at hand) the best compromise between large area and depth, good spectral resolution and coverage, in order to produce an optimum output in terms of redshift and SED accuracy. The ALHAMBRA survey is a deep photometric survey using 20 contiguous, equal-width, mediumband optical filters from $3500 \AA$ to $9700 \AA$, plus the three standard broadband $\left(J H K_{s}\right)$ NIR filters. The total area surveyed by ALHAMBRA will be $4 \mathrm{deg}^{2}$, being therefore placed halfway between traditional imaging and spectroscopic surveys.

By design, the ALHAMBRA survey will provide precise $(\Delta z<0.03(1+z))$ photometric redshifts and SED classification for several hundred thousand galaxies and active galactic nuclei (AGNs), allowing for different kinds of analysis regarding populations and structures, and their evolution in time. The details of the project, including simulations and expected results, and all the related aspects are described in the ALHAMBRA book that can be found at http://www.iaa.es/alhambra. Thanks to the unbiased nature of this survey (i.e., it was neither designed to detect a given class of objects nor to be precise only in some fixed spectral window), important problems other than cosmic evolution can be addressed. These include the study of stellar populations in the galactic halo, the search for peculiar stellar objects, ranging from very cold stars to blue stragglers, and the possible detection of debris from galactic satellites in the Milky Way halo. Moreover, the large surveyed volume and the ability to finely discriminate between different SEDs will permit the serendipitous detection of objects that could be classified as exotic or rare. This broad category includes very high redshift galaxies $(\approx 2500$ objects at $z>5$, with $\Delta z<0.01$, expected from scaled Hubble Deep Field (HDF) observations) and quasistellar objects (QSOs).

The observations are being carried out with the $3.5 \mathrm{~m}$ telescope of the Centro Astronómico Hispano-Alemán (CAHA), Calar Alto Observatory (Almería, Spain) and the wide-field imagers in the optical Large Area Imager for Calar Alto (LAICA) and in the NIR (Omega-2000). The collected data render possible the study of many different astronomical problems in a self-contained way and will provide very interesting targets for individual studies with large-size telescopes.

A separate article (Benítez et al. 2008) deals with the selection of the optical filters and the optimization of their characteristics to maximize the spectral information, while in this work we present the main characteristics that specifically define the ALHAMBRA survey, including some preliminary results from the data we have already accumulated.

This paper is organized as follows: in Section 2 we present the project implementation and its present status, and in Section 3 the first, preliminary results. We compare the ALHAMBRA survey with other surveys in Section 4, and our conclusions are presented in Section 5.

\section{SURVEY DESIGN, IMPLEMENTATION, AND STATUS}

\subsection{Description of the Filter System}

The idea to use photometric information to determine the redshift of faint sources was first proposed by Baum (1962), and later re-launched by Loh \& Spillar (1986), Koo (1986), and Connolly et al. (1995) as a poor person redshift machine. Its importance and adequacy to produce relevant data for different cosmological analyses was recognized after, among others, the works by Lanzetta et al. (1996), Connolly et al. (1997), and Fernández-Soto et al. (1999), on the HDF. Nowadays, many if not most, of the surveys that have already been completed or are under development, include the use of broadband filters and photometric redshift techniques.

Hickson et al. (1994) were the first to discuss the possibility of using a set of medium-band filters to continuously cover a large spectral range, and produce photometric data that could be considered equivalent to a very low resolution spectrum for each detected object. However, in that work there was no discussion of the number and kind of filters that would be needed in order to optimize the output in terms of $z$ and SED accuracy for a given instrumental setup and observing time. Later the surveys Calar Alto Deep Imaging Survey (CADIS; Meisenheimer et al. 1998) and Classifying Object by Medium-Band Observations17 (COMBO-17; Wolf et al. 2001, 2001, 2004; Bell et al. 2004) used a combination of broad- and medium-band filters with similar purposes. In particular, COMBO-17 employs the 


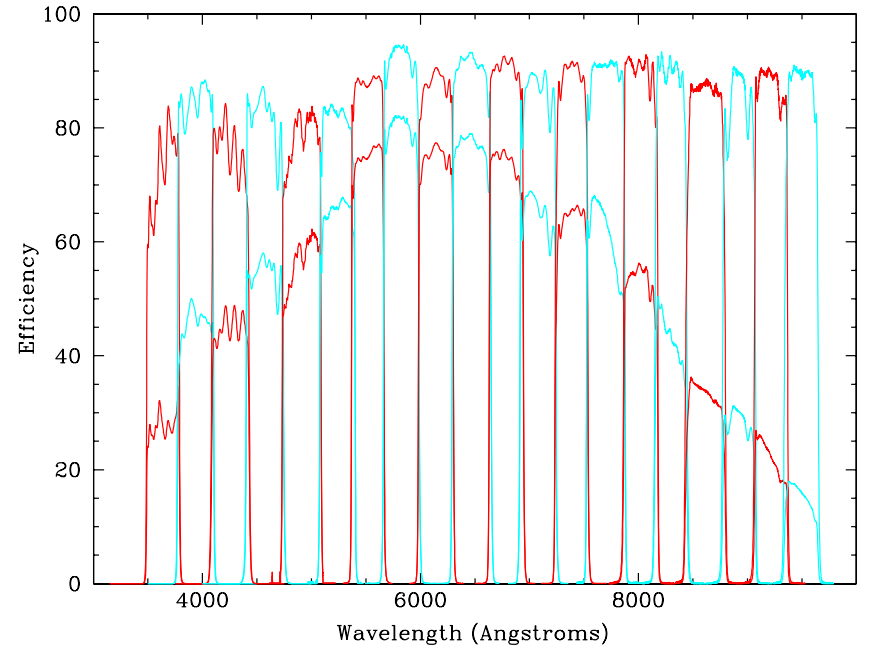

Figure 1. Transmission curves for one of the optical filter sets for the ALHAMBRA survey as measured in the laboratory. The effective total transmission (lower curve), after taking into account the quantum efficiency of the CCD detector, the atmosphere transmission (at Air Mass $=1.3$ ), and the reflectivity of the primary mirror of the Calar Alto $3.5 \mathrm{~m}$ telescope is also shown.

(A color version of this figure is available in the online journal.)

standard broadband filters (UBVRI) plus 12 narrow- or mediumband filters sampling several spectral domains between 4000 and $9200 \AA$. At the end, in this and similar surveys, the full spectral coverage is obtained via broadband filters. COMBO-17 has reached very good quality in getting photometric redshifts with an accuracy of $1 \%$ in $\Delta z /(1+z)$ at $R<21$. It covers an area of $1 \mathrm{deg}^{2}$. The degradation in quality at fainter magnitudes is planned to be compensated with the use of four redder filters $(Y, J 1, J 2$ and $H)$ in the MANOS-deep survey (or COMBO$17+4)$ covering a field of $0.77 \mathrm{deg}^{2}$.

The ALHAMBRA survey was designed to cover all the visible spectral domain with equally wide, contiguous mediumband filters to optimize its scientific output in terms of accuracy of the $z$ and SED determinations. By design, it is possible to detect, in addition to the overall SED, relatively faint emission lines. As already pointed out, the details are explained in a separate paper by Benítez et al. (2008), where different implementations were devised and analyzed to get the best solution for a given fixed total amount of observing time. The resulting optical filter system includes a total of 20 contiguous, medium-band, FWHM $=310 \AA$, top-hat filters, that cover the complete optical range from 3500 to $9700 \AA$. The optical coverage is supplemented with the standard NIR $J H K_{s}$ filters.

The filter set was designed with a series of restrictive requirements about spectral shape, homogeneity, and transmission in mind. In particular, all filters should have very steep side slopes, close to zero overlap in $\lambda$, a flat top, and transmissions in excess of $70 \%$. Given the particular disposition of the four detectors in the LAICA focal plane, four sets of filters had to be produced, in such a way that all of them were equivalent within strict limits. The complete set of $20 \times 4$ filters was manufactured by BARR Associates. They were confirmed to be within specifications at the laboratory of the Instituto de Astrofísica de Andalucía. The transmission curves are shown in Figure 1.

\subsection{Expected Redshift Precision and the NIR Filters}

For a survey with the characteristics of ALHAMBRA, the quality of the final results depends critically on the photometric errors and the adequacy of the templates used to compute

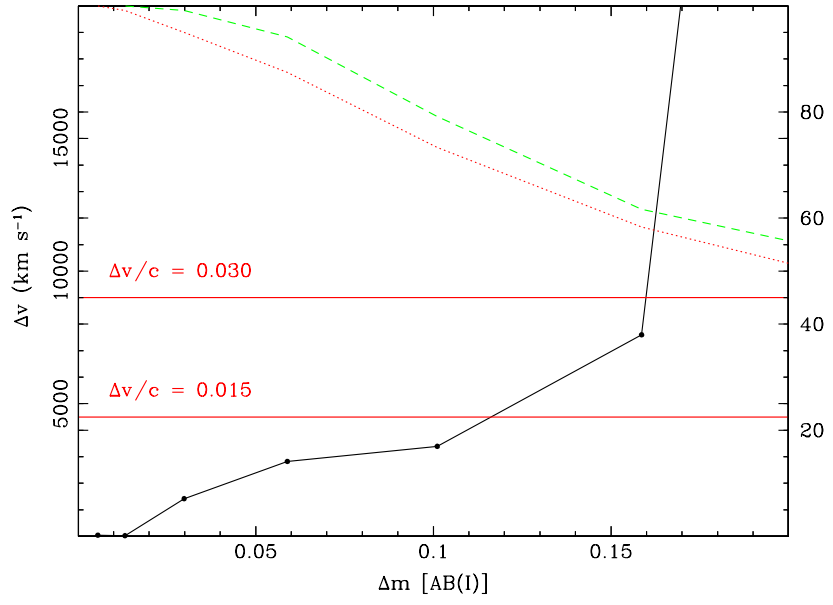

Figure 2. Estimates of the photometric redshift dispersion as a function of the photometric uncertainty in the $I$ band. The black dots and continuous line show the velocity uncertainty, while the red dotted (green dashed) line shows the percentage of objects with a given $I$-band uncertainty that have redshift residuals smaller than $\Delta z /(1+z)=0.015$ (0.030).

(A color version of this figure is available in the online journal.)

photometric redshifts. To test the first aspect, we have created a grid of galaxy spectra using the templates presented in Benítez (2000), with redshifts between 0 and 5.0, and absolute $K_{s}$-band magnitudes ranging from -23 to -16 . All these galaxies have been "observed" through the full ALHAMBRA photometric system, with noise added to the observed fluxes according to the properties of our data. Then each galaxy's ALHAMBRA photometric redshift was measured via the same code used by Fernández-Soto et al. (1999), and the offset between the original and the calculated values of the redshift was obtained. We then binned the objects according to their photometric $I$-band magnitude uncertainty, and estimated the dispersion around the correct redshift value for each bin. The results are presented in Figure 2, where it can be seen that we obtained an excellent redshift accuracy $(\Delta z /(1+z)<0.03)$ even for objects with magnitude errors as large as $\triangle A B(I) \approx 0.15$; the error we measure for typical objects is at $A B(I) \approx 24.5$. Moreover, the percentage of all those objects that actually have redshift residuals smaller than that is larger than $50 \%$. Recall that the redshift error distribution is strongly non-Gaussian, with long tails and second peaks due to the so-called "catastrophic errors." Of course, this analysis does not include the possible systematic effects induced by the choice of the template set.

Inclusion of the NIR information can significantly improve the determination of the redshift in some cases. In particular, as pointed out by many authors using photometric redshift techniques in deep surveys (e.g., Coe et al. 2006), the use of NIR filters can help to break the degeneracy between low-redshift $(z \approx 0.5)$ and high-redshift $(z \approx 3)$ galaxies. The reason behind this degeneracy is the possible confusion between the Balmer and Lyman breaks, which are the most salient features of the respective SEDs. In the absence of any infrared information, it is not possible to determine the slope of the rest-frame red end of the spectrum, the range that can in fact tell the difference between both families. Each one of the left-side panels in Figure 3 explicitly shows the degeneracies between the different spectral types and redshifts. For each of the six galaxy types used in the exercise (rows and cells) and every redshift (from $z=0$ to $z=8$ in both axes in each box), the points mark the types that degenerate with it in color space-for this particular figure, a magnitude "thickness" $\Delta m=0.2$ has been chosen, 

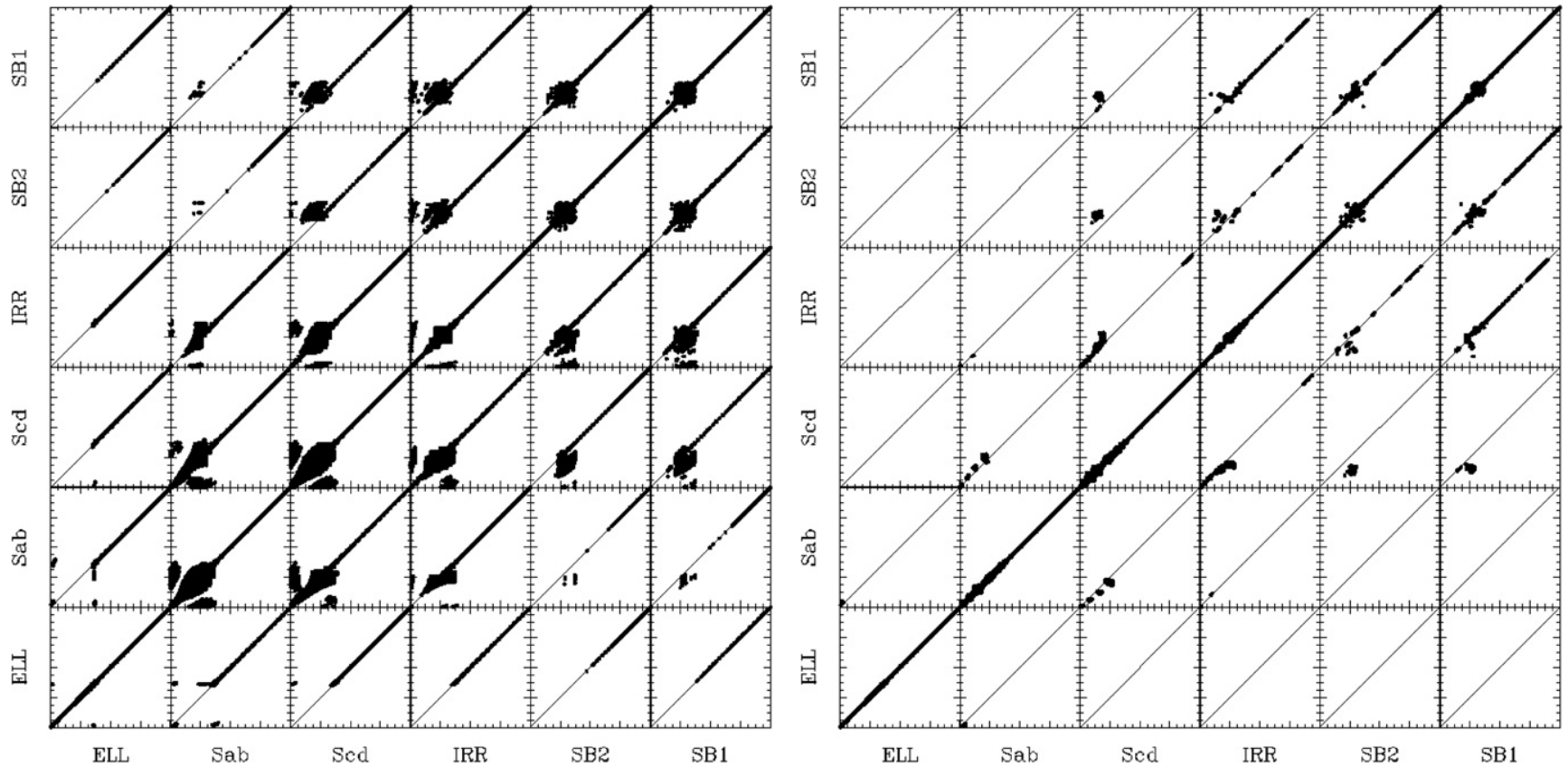

Figure 3. Theoretical degeneracies in type and redshift expected for galaxies in the ALHAMBRA survey measured to an accuracy of $0.2 \mathrm{mag}$ in all filters. The left panel shows the case where no infrared information is available, and the right panel corresponds to the case where the NIR information is included. Each of the $6 \times 6$ subpanels corresponds to a $z_{1}$ (photometric redshifts) vs. $z_{2}$ (actual values) diagram with redshifts ranging from 0 to 8 .

so that any two galaxy spectra that differ in less than $\Delta m$ in all the ALHAMBRA filters considered have been viewed as degenerate, and hence indistinguishable.

It can be seen in the right-side panel how (for the $\Delta m=$ 0.2 case) the presence of NIR data eliminates most of the degeneracies between low and high redshift, and sharply separates the elliptical, Sab, and Scd galaxies from the rest and from each other, leaving only some residual degeneracy between the three bluest types. Of course, the infrared information also adds greatly to the scientific content of the survey, via the more direct relation existing between the galactic mass and the infrared luminosity.

Our preliminary results (see the next section) show us that the simulations above should not be far off the mark; our $I$-band photometric errors are $<10 \%$ up to $I \approx 24$, with expected redshift errors $\delta z /(1+z) \leqslant 1.5 \%$. We also expect that most of the objects up to $I \leqslant 25$ will have photometric redshift errors $<3 \%$.

\subsection{The Survey Area}

It is well known that astronomical objects cluster on the sky on different scales. The clustering signature contains a wealth of information about the structure-formation process. A survey designed to cope with the cosmic variance and to describe and understand the clustering needs to probe as many scales as possible, up to the homogeneity scale. So, on one hand, searching contiguous areas is important in order to cover smoothly the smallest scales where the signal is stronger and to obtain an optimally-shaped window function. On the other hand, measuring a population of a certain volume density is a Poissonian process with an associated variance and, in principle, one would obtain different densities of the same population when measuring at different places. The variance in those measurements is dictated by the volume density of the population under study, the volume searched, and the clustering of the population. In order to beat down this cosmic (or, technically, sampling) variance, one needs to survey independent volumes. So, all in all, a balance must exist between probing contiguous and independent areas.

For a survey aiming at resolving a number $N_{\text {st }}$ of SED-types, the sampling errors in any counting statistical measure will be

$$
\sigma_{s}=K \sqrt{\frac{N_{\mathrm{st}}}{N_{f} \Delta z}},
$$

where $N_{f}$ is the number of fields of a given area and $\Delta z$ is the redshift interval to be resolved. To get a quantitative evaluation of this statistical noise, we have used the SDSS local luminosity function, which yields, with a plausible parameterization of the evolution, a redshift distribution similar to that obtained from the $\mathrm{HDF}$ at our magnitude limit. Expressing $N_{f}$ as the number of $1 \mathrm{deg}^{2}$ fields, we find $K$ in the range $0.01-0.02$ for redshift between 0.3 and 2 . Therefore, if we intend to have relative sampling errors no larger than a few percent at any redshift, with a redshift resolution better than $\Delta z=0.05, N_{f}$ should be similar to $N_{\text {st }}$, which results in a total field of a few square degrees.

On the technical side, we had to consider the geometry of the imager LAICA, with four $4 k \times 4 k$ CCDs, arranged in a $2 \times 2$ mosaic with the gap between two adjacent (vertical and horizontal) CCDs being almost the same size of the CCD itself. Thus, one pointing corresponds to four patches of $15^{\prime} .4 \times 15^{\prime} .4$ over a total field of $44^{\prime} .4 \times 44^{\prime} .4$, such that with four pointings, a total, contiguous area of $1 \mathrm{deg}^{2}$ is covered. ${ }^{12}$ This geometry imposes a minimum contiguous area patch of $1 \mathrm{deg} \times 0.25 \mathrm{deg}$ and produces two such strips in two pointings. The Omega2000 camera used for NIR observations ${ }^{13}$ covers a field of view equivalent to one of the LAICA CCDs.

Considering the sampling constraints together with the technical characteristics of the detectors, the expected efficiency of the atmosphere-telescope-imager, and the amount of available

\footnotetext{
12 http://www.caha.es/CAHA/Instruments/LAICA/index.html.

13 http://www.mpia-hd.mpg.de/IRCAM/O2000/index.html.
} 
Table 1

The ALHAMBRA-Survey Fields

\begin{tabular}{lcccccc}
\hline \hline Field Name & R.A. (J2000) & Decl. (J2000) & $100 \mu \mathrm{m}$ & $E(B-V)$ & $l$ & $b$ \\
\hline ALHAMBRA-1 & 002946.0 & +052528 & 0.83 & 0.017 & 113 & -57 \\
ALHAMBRA-2/DEEP2 & 022832.0 & +004700 & 1.48 & 0.031 & 166 & -53 \\
ALHAMBRA-3/SDSS & 091620.0 & +460220 & 0.67 & 0.015 & 174 & +44 \\
ALHAMBRA-4/COSMOS & 100028.6 & +021221 & 0.91 & 0.018 & 236 & +42 \\
ALHAMBRA-5/HDF-N & 123500.0 & +615700 & 0.63 & 0.011 & 125 & +55 \\
ALHAMBRA-6/GROTH & 141638.0 & +522505 & 0.49 & 0.007 & 95 & +60 \\
ALHAMBRA-7/ELAIS-N1 & 161210.0 & +543000 & 0.45 & 0.005 & 84 & +45 \\
ALHAMBRA-8/SDSS & 234550.0 & +153450 & 1.18 & 0.027 & 99 & -44 \\
\hline
\end{tabular}

Note. We include together with the name a reference to overlaps with other surveys of interest.

time for the project, we finally decided to cover two such strips in each of the eight selected fields, a total of $4 \mathrm{deg}^{2}$ to ensure a large enough area coverage and good sampling.

The fields were selected taking their low extinction as first and basic criterion, as measured in Schlegel et al. (1998). Then, within the lowest extinction patches, we tried to identify those containing neither bright sources nor conspicuous structures. We decided that a significant overlap with other surveys would be an asset, to ensure the possible cross-checking of our results and maximum complementarity, in particular regarding the largest coverage in wavelength. The selected fields are listed in Table 1 . The $100 \mu \mathrm{m}$ emission and $E(B-V)$ values quoted in the table are the median for the whole $1 \mathrm{deg}^{2}$ field centered at the given position. Only ALHAMBRA-1 is new in the sense of having no overlap with other surveys.

At the time of the writing of this article (2008 February), we had already obtained about $64 \%$ of the NIR data and about $35 \%$ of the visible data. The NIR observations with Omega-2000 were started in 2004 August, whereas we were only able to begin observations with LAICA in 2005 September. We expect to complete our observations by 2010 .

We will discuss below in Section 3 some preliminary results obtained with our first complete $15^{\prime} \times 15^{\prime}$ pointing, including observations with all the ALHAMBRA filters.

\subsection{The Calibration Strategy}

As mentioned above, we have already completed the first LAICA pointing in all the filters. In the quoted ALHAMBRA book we have presented the simulations we performed to design the survey and to find the expected depth of the data for the strategy chosen. We are now in the position of checking the quality of the data delivered by the survey, the validity of our simulations, and the adequacy of our observational strategy.

Indeed, a crucial aspect to do this analysis is the photometric calibration of the data. This is a particularly demanding aspect of the project. Apart from other considerations, it has been shown that the reliability of the photometric redshifts depends critically on the photometric accuracy (Coe et al. 2006). This aspect is particularly acute in our case since we are in fact introducing a new photometric system in the optical domain. Thus, special care is needed to anchor it to existing photometric systems and to primary calibrators.

To define the reference fluxes and magnitudes of the ALHAMBRA photometric system we have chosen a set of primary stars from the lists by Oke \& Gunn (1983), Oke (1990), Massey \& Gronwall (1990), and Stone (1996), together with the four fundamental calibrators adopted by the HST. The list includes the standard star BD $+17^{\circ} 4708$, the primary calibrator of the SDSS system.
We give here a short account of calibration procedure, whereas the complete details will be presented in a forthcoming article. The devised procedure starts with the selection of stars in our frames that have SDSS photometry. These will play the role of secondary standards. We plan to obtain spectrophotometric observations of all these stars, calibrated with respect to those primary standards. The spectra will then be fitted by stellar models and the ALHAMBRA system colors will be obtained from the fitted models by integration over the filters. We will also compare the synthetic $u^{\prime} g^{\prime} r^{\prime} i^{\prime} z^{\prime}$ colors with the SDSS values to check the consistency between the systems. In this way, we expect to have a calibration that is accurate to the $2 \%$ level or better. The comparison between photometric and spectroscopic redshifts for the galaxies with spectroscopic observations will also be used as a check of the calibration of the ALHAMBRA data (Coe et al. 2006).

We will set the ALHAMBRA magnitudes on the AB system (Oke \& Gunn 1983),

$$
A B_{v}=-2.5 \log f_{v}-48.60
$$

where $f_{v}$ is the flux per unit frequency from an object in erg s $\mathrm{s}^{-1}$ $\mathrm{cm}^{-2} \mathrm{~Hz}^{-1}$.

The magnitudes will be defined with reference to their spectrophotometric data by adopting

$$
m=-2.5 \log \frac{\int_{F} f(v) \mathrm{S}_{F}(v) \mathrm{d}(\log v)}{\int_{F} \mathrm{~S}_{F}(v) \mathrm{d}(\log v)}+\text { Cte }
$$

where $S_{F}(v)$ is the transmission curve corresponding to the atmosphere-telescope-filter-detector combination.

This is in fact the usual way to calibrate narrow-band images when the photometric system is not previously defined (see, for example, Márquez \& Moles 1996). The SDSS Consortium has also adopted this strategy to define their own photometric system (Fukugita et al. 1996; Smith et al. 2002).

At the time of writing the present paper we have not yet completed the observations of secondary stars to get the final calibration. In order to get a preliminary calibration that would be robust and accurate enough to allow the analysis of the main aspects of the survey in terms of depth and reliability, we have implemented a different procedure. Briefly, what we have selected are 228 flux calibrated stellar spectra from the Hubble's Next Generation Spectral Library (NGSL; Gregg et al. 2004) that cover a wide range of physical properties. From these spectra synthetic colors were obtained in both SDSS and ALHAMBRA systems and the first set of transformation equations between the two systems were obtained. The results are given in Table 2. 
Table 2

The Calibration Relations

\begin{tabular}{|c|c|c|c|c|c|c|}
\hline Filter & Orig. & $u$ & $g$ & $r$ & $i$ & $z$ \\
\hline 01 & -0.0208 & 0.9314 & & & 0.0690 & \\
\hline 02 & 0.0257 & 0.3626 & 1.3321 & -0.7066 & & \\
\hline 03 & -0.0467 & 0.1442 & 1.3099 & -0.4560 & & \\
\hline 04 & -0.0391 & & 1.0607 & & & -0.0587 \\
\hline 05 & 0.0147 & & 0.6488 & 0.5480 & & -0.1961 \\
\hline 06 & -0.0336 & & 0.6341 & 0.3667 & & \\
\hline 07 & -0.0192 & & 0.3199 & 0.7865 & -0.1056 & \\
\hline 08 & -0.0057 & & 0.0604 & 1.1792 & -0.2391 & \\
\hline 09 & -0.0050 & & & 1.1009 & -0.1008 & \\
\hline 10 & 0.0067 & & & 0.7279 & 0.2720 & \\
\hline 11 & 0.0188 & & -0.2000 & 1.1309 & 0.0682 & \\
\hline 12 & 0.0156 & & -0.0876 & 0.6046 & 0.4811 & \\
\hline 13 & -0.0284 & & 0.0839 & -0.1523 & 1.0693 & \\
\hline 14 & 0.0208 & -0.0178 & & & 1.0177 & \\
\hline 15 & 0.0247 & & & -0.2473 & 1.2463 & \\
\hline 16 & 0.0372 & & & -0.3725 & 1.3717 & \\
\hline 17 & 0.0161 & & & & 0.1777 & 0.8207 \\
\hline 18 & -0.0528 & & & & -0.0383 & 1.0413 \\
\hline 19 & -0.0199 & & & & -0.1200 & 1.1207 \\
\hline 20 & 0.0186 & & & & -0.2436 & 1.2433 \\
\hline
\end{tabular}

Note. The numbers are the coefficients of the relation between any given ALHAMBRA magnitude and the SDSS values (blanks for null coefficients).

Then we have applied these equations to a set of stars with accurate SDSS and ALHAMBRA instrumental magnitudes to obtain the zero point of the photometric calibration. The zero points found in this way are accurate at the level between 5 and $10 \%$.

The NIR data have been calibrated through the 2MASS catalog stars present in our frames. The rms of the calibration is below 0.04 mag in all three bands.

\subsection{Observing Strategy}

As was mentioned in Section 2.1, the ALHAMBRA filter set was devised following detailed simulations in order to optimize the quality and number of photometric redshifts for galaxies in our fields. Similarly, the exposure times per filter were defined in such a way that the expected magnitude limits in each one would be as homogeneous as practically possible. This driving principle, combined with a minimum exposure time of $2.5 \mathrm{ks}$ per filter (due to the logistics of the observation procedure), and the known fact that our system is less efficient in the red end of the spectrum, prompted us to divide the total LAICA time per pointing $(100 \mathrm{ks})$ in the way presented in Figure 4. Thus, the target $A B=25$ limit would be reached or even exceeded for all filters bluewards of $\approx 8500 \AA$, and redwards from there, the decreasing efficiency makes the limit magnitude drop until $A B \approx 23$ at $\approx 9500 \AA$. In the NIR, because of previous experience with Omega-2000, we decided to divide the available $15 \mathrm{ks}$ per pointing equally into the $J, H, K_{s}$ filters, aiming to reach $J=22, H=21, K_{s}=20$ (in the Vega-based system). These expectations will be compared to the real data in Section 3.

Our image quality limit to consider the data as "scientifically valid" is, in real time at the telescope dome, a value of the seeing which should be $<1^{\prime \prime}$.4. However, the seeing, as well as the transparency, is measured a posteriori during the pipeline reduction, and the individual images are kept or eliminated from the final combination based on a more complex quality assessment that will be presented elsewhere.

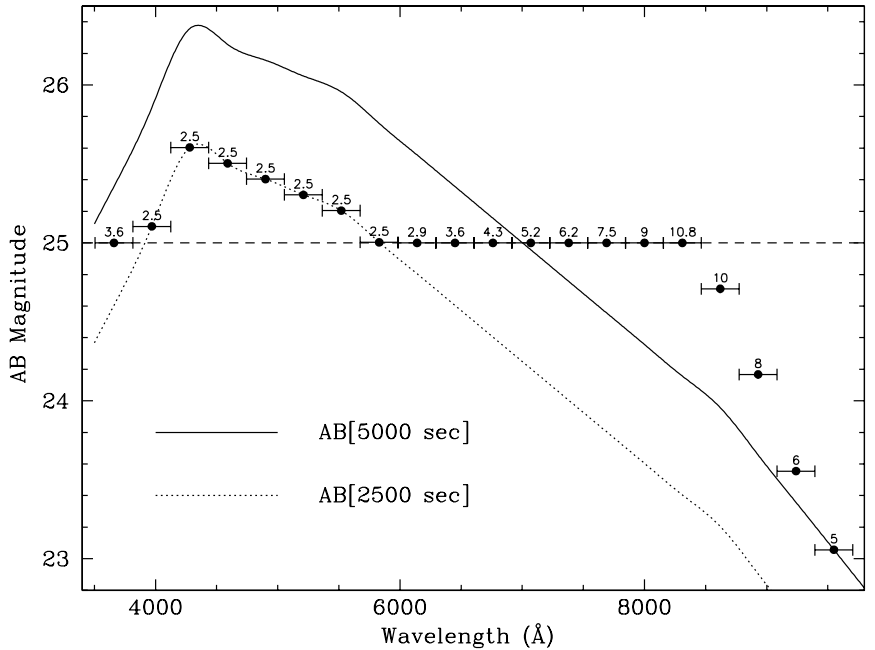

Figure 4. Expected limit magnitudes for the ALHAMBRA filters, at $\mathrm{S} / \mathrm{N}=5$, estimated for our survey. The exposure time per filter (in ks) is given for every filter.

\subsection{Data Reduction}

For the reduction of the images, two different pipelines have been used. In the case of the LAICA data, the preliminary standard steps of overscan correction and flat-fielding were performed. After that, the illumination correction using smooth superflats was applied. In the optical filters redder than $7000 \AA$, the images suffer from a substantial fringing pattern that is removed using the procedure described in Alcala et al. (2002). This fringing pattern contributes less than $2 \%$ to the flat fields, so we have not corrected the flat fields at this stage.

In the case of the NIR filters, we first removed the dark current frames and divided by a normalized superflat that was constructed by combining the science images. An additive pupil ghost is present in the superflat images, that is more prominent in the $J$ band ( $~ 5 \%$ contribution). The pupil ghost is fitted in the normalized flat-field images using the mscpupil task in the IRAF MSCRED package (Valdes 2002). The adjusted pupil pattern $\left(p_{i j}\right)$ is removed from the flat field dividing it by $\left(1+p_{i j}\right)$, and is subtracted from the individual images after scaling it by the median background level. After having flat-fielded the images, the sky structure of each individual image is removed with the XDIMSUM package (Stanford et al. 1995) using the sky image constructed with the median of the six closest images, that in case of the $J, H, K_{s}$ filters correspond to timescales of 480,360 , and $276 \mathrm{~s}$, respectively. Before removing the background, the sky image is median filtered using a $5 \times 5$ window in order to reduce its noise. During this process masks for each individual image with the location of the cosmic rays are also created. The cosmic rays and bad pixels are fixed to proceed with the next steps.

At this stage, the sky level is measured, and SExtractor (Bertin \& Arnouts 1996) is used to obtain an initial estimate of the FWHM of each image, and the number of sources above a given signal-to-noise ratio $(\mathrm{S} / \mathrm{N})$. The relative transparency of each image is measured using high $\mathrm{S} / \mathrm{N}$ stars. These numbers are used to remove bad images or images out of the survey requirements. After that, the astrometry of a reference image is calibrated using the USNO-B1.0 (Monet et al. 2003) catalog. The external astrometric rms is $\sim 0.12$ arcsec in each axis. The remaining images are calibrated internally, in a first iteration using the reference image in the filter. After completing an ALHAMBRA 

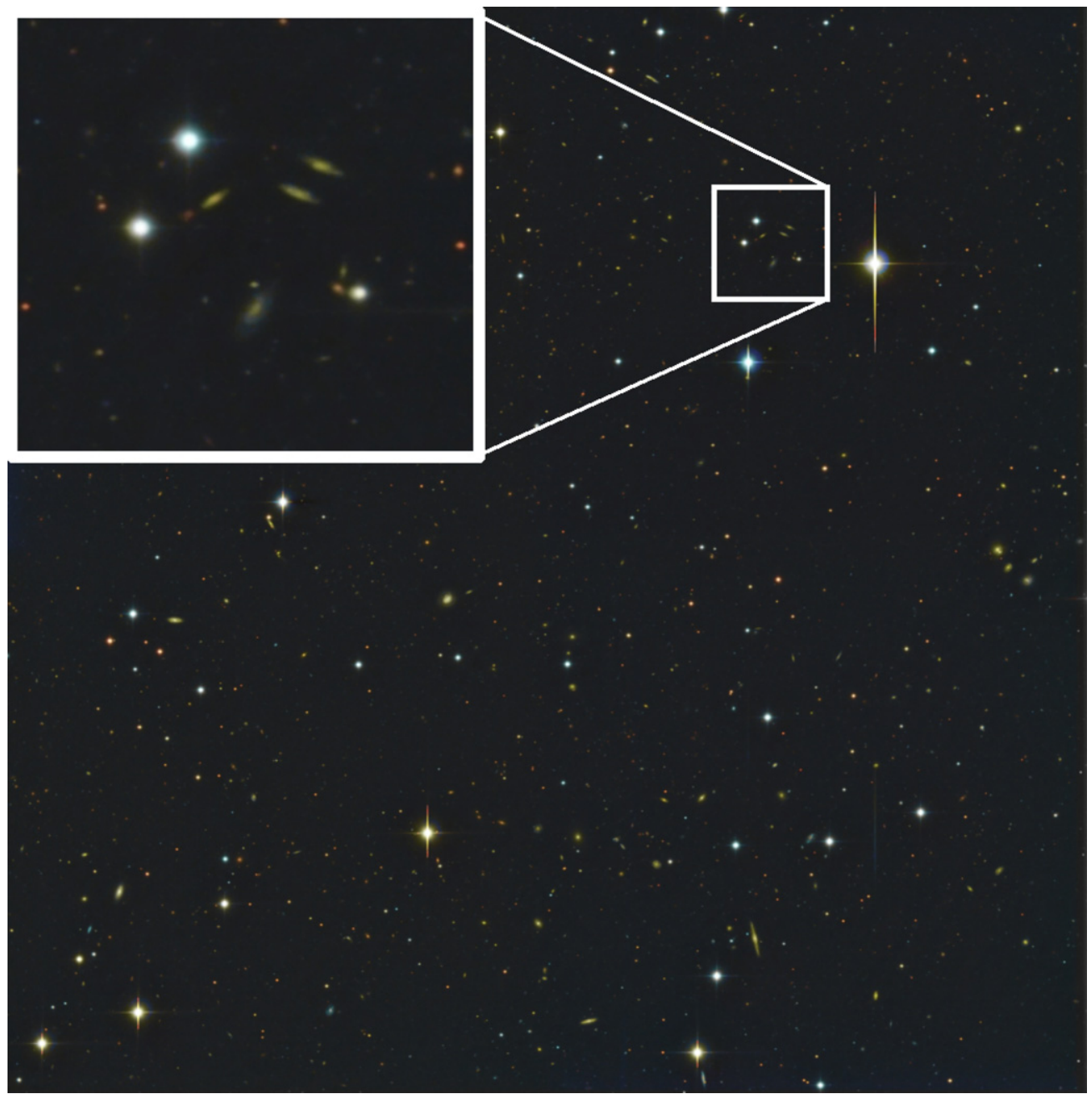

Figure 5. The first complete pointing of the ALHAMBRA-8 field in a square region of 15 arcmin side. This color image has been created making use of data from 14 out of the 23 filters. In the insert, corresponding to a region of about 1.5 arcmin, a small group of galaxies can be seen.

pointing a deep image constructed using images with good FWHM and transparency in several selected filters will be created and used afterwards to calibrate the internal astrometric solution. For this purpose we have used our own algorithm to match the image sources with those in the external catalog, and the IRAF ccmap task to obtain an order 3 polynomial solution. The internal astrometric solution rms is $\sim 0.03$ arcsec in each axis for the LAICA images and $\sim 0.05$ arcsec in the Omega2000 ones. Finally, the astrometry of each individual image is re-calibrated internally with the reference image using Scamp ${ }^{14}$ obtaining similar results. For image combination, Swarp ${ }^{15}$ is used. This software takes into account the distortion pattern present in the wcs headers, and allows the user to obtain resampled images with a different pixel size, in the desired sky projection. Also the previously computed relative transparency is used to uniform the zero points of the individual images. In our process, before performing the final combination using Swarp, the resampled images are used to apply a pixel rejection algorithm in order to improve the cosmic ray rejection and bad pixel masks. In the case of the Omega-2000 images, with a roughly double pixel size, we also require a final image with the LAICA pixel scale. The final images for each ALHAMBRA

\footnotetext{
14 http://terapix.iap.fr.

15 http://terapix.iap.fr.
}

pointing in the 23 filters are registered within the internal astrometric solution rms.

\section{SURVEY PERFORMANCE: PRELIMINARY RESULTS}

The ALHAMBRA survey and filter system design were preceded by realistic, thorough simulations presented in Benítez et al. (2008) and in the ALHAMBRA book. Recently, we completed the observations of the first pointing in the $20+$ 3 filters, comprising four times a 15.4 $\times 15^{\prime} .4$ field, and we are thus able to compare the performance of the survey with our expectations.

Figure 5 shows a color image corresponding to one of the CCDs of the first complete pointing in the ALHAMBRA-8 field (see Table 1). An enlargement of this image of approximately 1.5 arcmin side, where a small group of spiral galaxies can be seen, is shown as an insert in the same figure.

These data allow us to go from simulations to actual measurements, and describe several key characteristics of the survey; others will have to be complemented with simulations until we carry out follow-up observations, primarily due to the paucity of available spectroscopic redshifts in this particular field.

\subsection{Photometric Depth}

The data reduction procedure involves the standard steps, including accurate flat-fielding and defringing (for the red filters) 


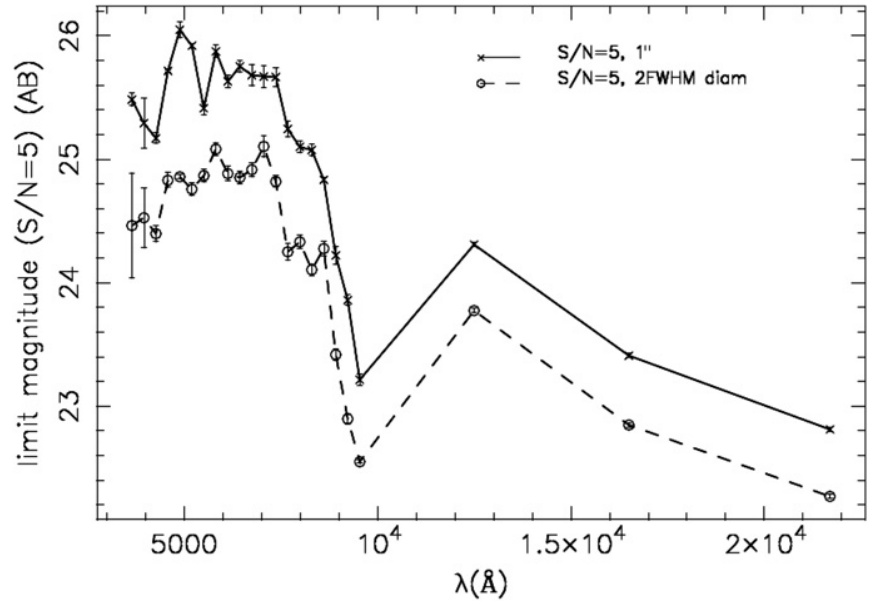

Figure 6. The limit $A B$ magnitude, at $\mathrm{S} / \mathrm{N}=5$ in each filter, for $1^{\prime \prime}$ square aperture and for an aperture of $2 \times$ FWHM diameter. The total observing time per pointing was fixed to $100 \mathrm{ks}$ for the 20 optical filters, and to $15 \mathrm{ks}$ for the NIR bands.

and precise astrometry. SExtractor, with just standard settings for the different parameters, was used to obtain the photometry of the detected objects that we discuss here. The pipelines and procedures will be described in a separate paper, and will be made publicly available. Then the data were photometrically calibrated following the procedure outlined in the previous section.

The ALHAMBRA filter system was primarily designed to obtain the best determination of $z$ and SED for a fixed amount of observing time per pointing $(100 \mathrm{ks})$. We estimated the expected limit magnitudes taking into account the average extinction in Calar Alto, the performance of the $3.5 \mathrm{~m}$ telescope-LAICA system for airmass $=1.3$, a final image quality of FWHM $\approx 1^{\prime \prime} .2$ and the measured filter+CCDs transmission curves. The goal was to get homogeneous magnitude limits for as many filters as possible, with the restriction that the minimum exposure time per filter should not be less than $2.5 \mathrm{ks}$. We expected to reach $A B \leqslant 25(\mathrm{~S} / \mathrm{N}=5$, point-like source) for 16 filters ranging from $3500 \AA$ to $8500 \AA$. In the case of the four reddest filters (close in wavelength to the $z$ band) we could obtain decreasingly lowerlimit magnitudes, down to $A B=23.4$ in the last filter centered at $9550 \AA$ (see the quoted ALHAMBRA book and Moles et al. 2005).

To ascertain in a quantitative way the actual depth reached in each of the filters, we have defined the limiting magnitude as that corresponding to the rms within a $1 \operatorname{arcsec}^{2}$ aperture, at the $\mathrm{S} / \mathrm{N}=5$ level. The values we found are plotted in Figure 6 . As can be seen, we are actually reaching the expected limits in all the filters, including the reddest ones. We also show in the same figure the magnitude limit values for a $2 \times$ FWHM aperture, which may represent a more realistic integration area for typical flux measurements.

A complementary way to illustrate the depth of the data is to consider the number counts in each band. In Figure 7, we show the preliminary number count histograms in the 20 ALHAMBRA optical filters. The counts have been normalized to unit magnitude and unit area $\left(\mathrm{deg}^{2}\right)$. No corrections due to completeness or spurious detection have been applied. The histograms indicate that we are detecting sources at $A B \leqslant 25$ in all the filters from the bluest to about $8000 \AA$. The limiting magnitude then becomes brighter, and we end up with $A B \sim$ 23 at $9500 \AA$.

Moreover, to enable comparison with broadband and spectroscopic surveys (see later), we have synthesized broadband filters from our medium-band ALHAMBRA filters. In Figure 8 we present the results for the $I^{*}$ (synthetic $I$ ) band. It can be seen that the survey is complete to $I^{*} \approx 25$ with errors $\approx 20 \%$ in the worst cases.
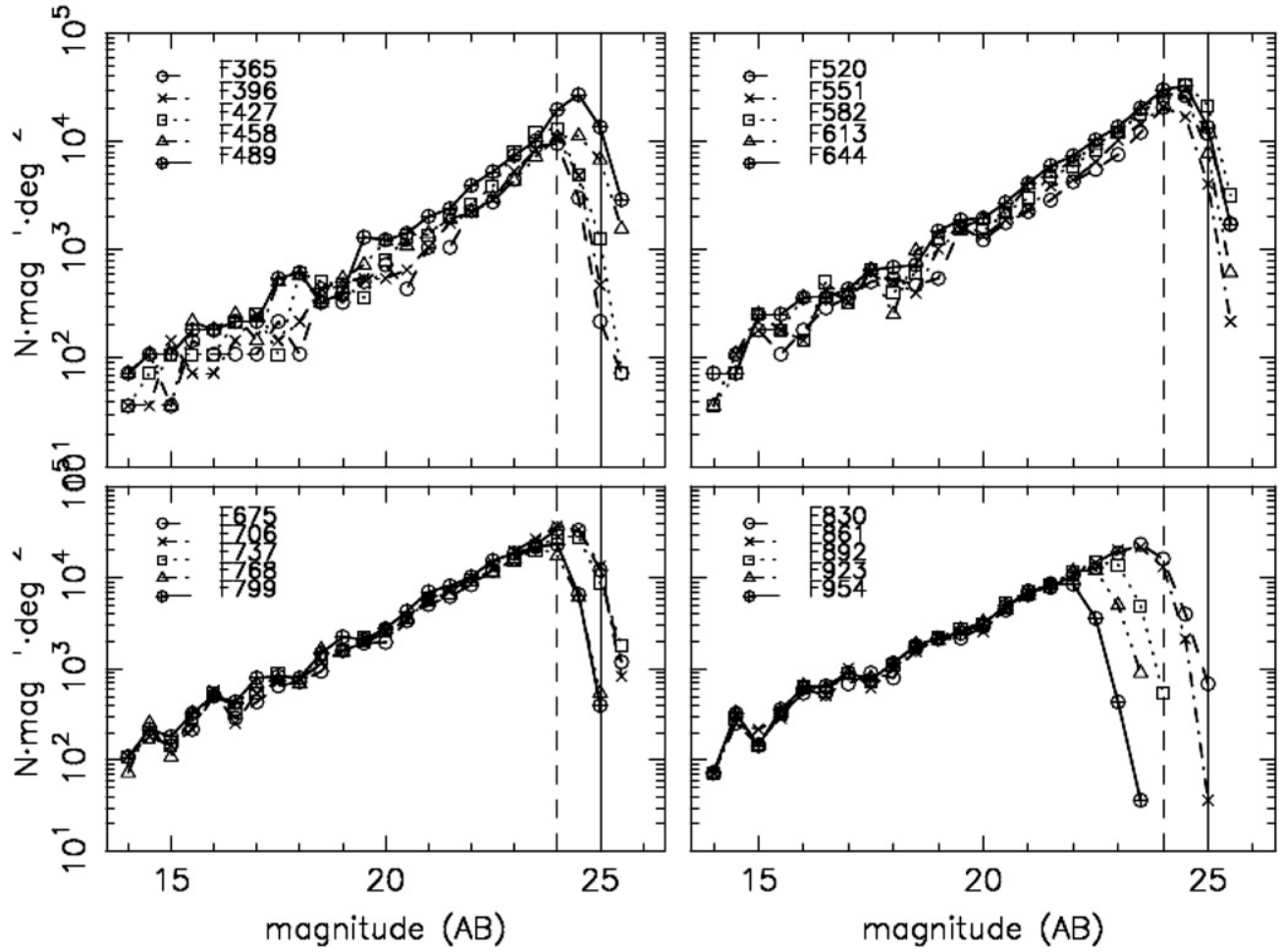

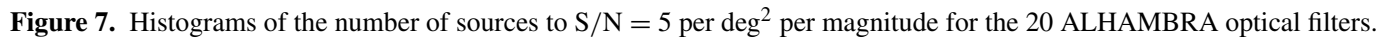




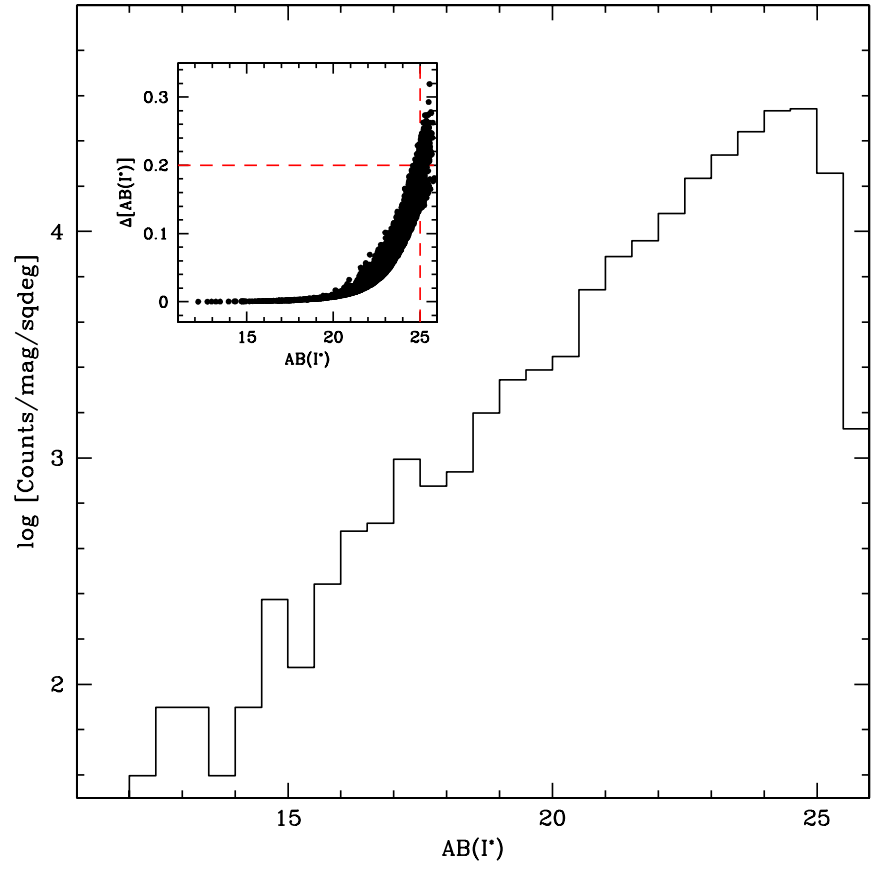

Figure 8. Counts as a function of isophotal magnitude in the synthetic $I$-band image. The completeness limit is close to $I(A B)=25$. In the inserted panel, the photometric errors are shown as a function of the isophotal $I$ magnitude.

(A color version of this figure is available in the online journal.)

In the NIR, based on the existing experience with Omega2000, we fixed the total exposure time to $5 \mathrm{ks}$ per filter, in order to reach $K_{s} \approx 20, H \approx 21, J \approx 22$ in the Vega system $(\mathrm{S} / \mathrm{N}=5$, point-like source). The limit magnitudes presented in Figure 6 and the histograms presented in Figure 9 show that these limits have been reached, and even exceeded. ${ }^{16}$ A more detailed analysis of the NIR galaxy counts will be presented in D. Cristóbal-Hornillos et al. (2008, in preparation).

\subsection{Photometric Redshift Depth and Accuracy}

The central goal of the ALHAMBRA survey is to measure with precision the observed photometry of as many objects as possible; this enables us to estimate accurate redshifts and spectral types. The use of a large number of filters, contiguous, and with minimal overlap among themselves provides a clear, unequivocal representation of the galaxy SED. This is well illustrated in Figure 10 which shows a small $(0.7 \times 0.7$ arcmin $)$ thumbnail image of an $A B(I) \approx 23$ object in the ALHAMBRA8 field in all 23 ALHAMBRA images and the synthetic $U^{*} B^{*} V^{*} R^{*} I^{*}$ filters, together with a calibrated ALHAMBRA "spectrum" of the object, a starburst with $z=4.23$.

The customary way of determining the photo- $z$ accuracy of a survey is by comparing estimates with a large sample of spectroscopic redshifts; unfortunately, the first field that we have completed only has SDSS spectroscopy available, which are too shallow and sparse for that purpose, and we plan to carry out spectroscopic follow-up observations for this and other fields.

The NASA Extragalactic Database contains nine galaxies with spectroscopic redshifts within the limits of our $15^{\prime} \times$ $15^{\prime}$ pointing, and all of them are SDSS galaxies (AdelmanMcCarthy et al. 2007), with redshifts in the range $z \lesssim 0.2$. Despite the inadequacy of this sample for statistical purposes,

${ }_{16}$ Recall that the magnitude difference ( $A B$-Vega) for the $J, H$, and $K_{s}$ filters is $\approx 0.9,1.5$, and 2.0 , respectively.

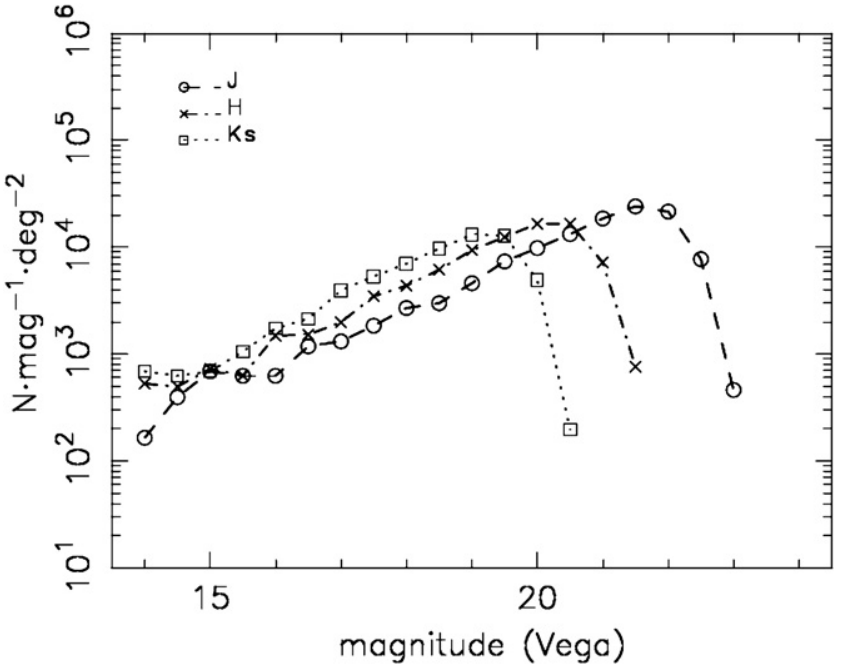

Figure 9. Histograms of the number of sources to $\mathrm{S} / \mathrm{N}=5 \mathrm{per} \mathrm{deg}^{2}$ per magnitude in the three NIR filters.

it allows us to give the reader a taste of the redshift accuracy of the ALHAMBRA survey (see Figures 11 and 12).

We are presently using three different codes to measure photometric redshifts, based on different methods, in order to eventually choose the most robust and accurate strategy.

(a) The first is the code developed by Fernández-Soto et al. (1999), based on galaxy template fitting with an extended Coleman et al. (1980) library comprising six templates.

(b) The second code is the BPZ program, as described in Benítez (2000), which uses a Bayesian magnitude-type prior and the empirically calibrated template library described in Benítez et al. (2004) complemented by two very blue starburst types (Coe et al. 2006), eight galaxy templates in total.

(c) The third method has been recently implemented by S. F. Sánchez et al. (2008, in preparation), and uses a synthetic library which also includes stellar spectra, AGN types, and dust reddening. The use of a synthetic library provides information about the metallicity, the star-formation history, and other characteristics of the object.

The empirically calibrated library of Benítez et al. (2004) has been shown to be precise enough to detect and calibrate photometric zero-point errors within $2-3 \%$ as those in the NIC3 observations of the Hubble Ultra Deep Field (see Coe et al. 2006). Using this calibration technique to correct the COMBO-17 photometry presented in Hildebrandt et al. (2008) reduces the photometric redshift error from $\delta z /(1+z)$ from $0.038 \pm 0.035$ to $0.001 \pm 0.023$. We therefore carry out a similar zero-point recalibration using the nine galaxies with spectroscopic redshifts. This recalibrated photometry is then fed to the three codes mentioned above. As expected, the agreement among them is good for bright objects, but somewhat breaks down at fainter magnitudes.

It is noteworthy that code (c) predicts the redshifts of the spectroscopic sample extremely well: its photometric error is $\delta z \approx 0.000 \pm 0.010$, which is close to the theoretical accuracy (see Figure 11 ). The BPZ software yields $\delta z \approx 0.002 \pm 0.014$, but it mistakes a reddened edge-on spiral at $z=0.09$ for a higher redshift, producing an outlier.

We will further test and refine our photometric redshift techniques, and the final strategy will probably include a combination of them, in order to make our measurements as 

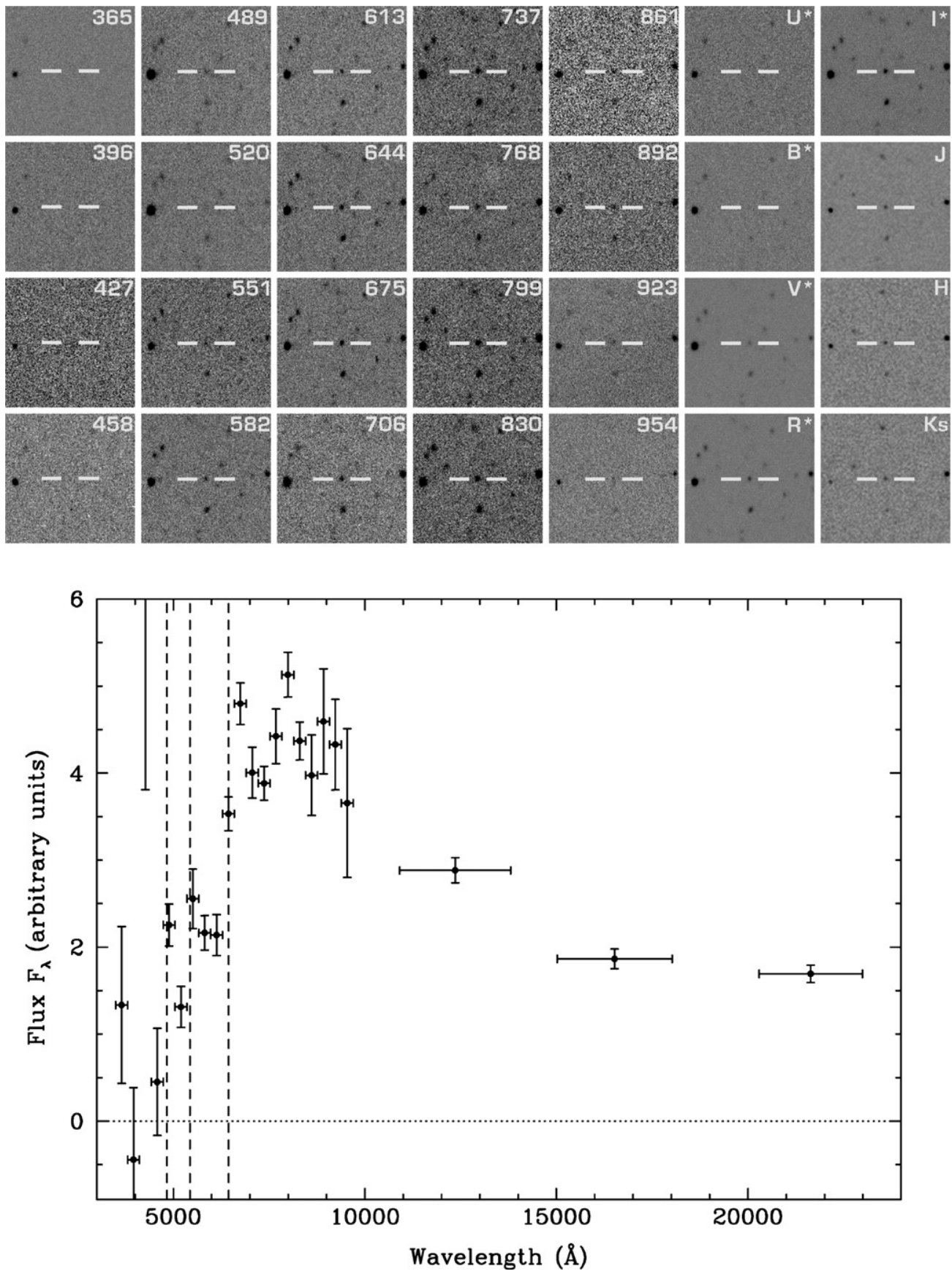

Figure 10. Upper panels: images through all 23 ALHAMBRA filters and the synthetic Johnson filters for a single object in the ALHAMBRA-8 field, marked with horizontal ticks. Each $0.7 \mathrm{arcmin}^{2}$ thumbnail is labeled with its wavelength in nanometers, or the filter name. Lower panel: the ALHAMBRA spectrum of the same object. The vertical dashed lines mark the putative positions of Lyman- $\alpha$, Lyman- $\beta$, and the Lyman limit, at the measured redshift $z=4.32$. Note that the $427 \mathrm{~nm}$ image and data point are noisier because we did not use the complete exposure time for this filter in this preliminary reduction.

robust as possible. As a token of the quality of the spectral information, we present the ALHAMBRA spectrum of one of the SDSS galaxies in Figure 12 (SDSS coordinates 356.331030, +15.479499 , redshift $z_{\mathrm{sp}}=0.113525$ ). Our best-fit redshift for this object is $z_{\mathrm{ph}}=0.12$. The magnitude offset between the SDSS spectrum and our photometry is present in the original data, and it must be due to the smaller flux falling within the SDSS spectrograph fiber. Our data, even with the preliminary calibration we are using at the moment, perfectly reproduce even some of the minor details in the spectrum.

In Figure 13 we present a mosaic of three-color galaxy images sorted according to redshift (lines) and luminosity (columns).
They span a large range in absolute magnitude (as measured in the band centered at $7370 \AA$ ), from brighter than $M(A B)=$ -24 , to $M(A B) \mathrm{s} \approx-16.7$. The figure illustrates the depth of this preliminary catalog and the redshift values that can be obtained.

One of the defining characteristics of any photometric redshift survey is its effective depth, i.e., the magnitude limit at which it is still possible to measure precise photometric redshifts. If the photometric redshifts are estimated using a Bayesian formalism, it is possible to define a quantity called "odds" which serves as a powerful quality indicator of the reliability and accuracy of a photometric redshift estimation (Benítez 2000, Benítez 


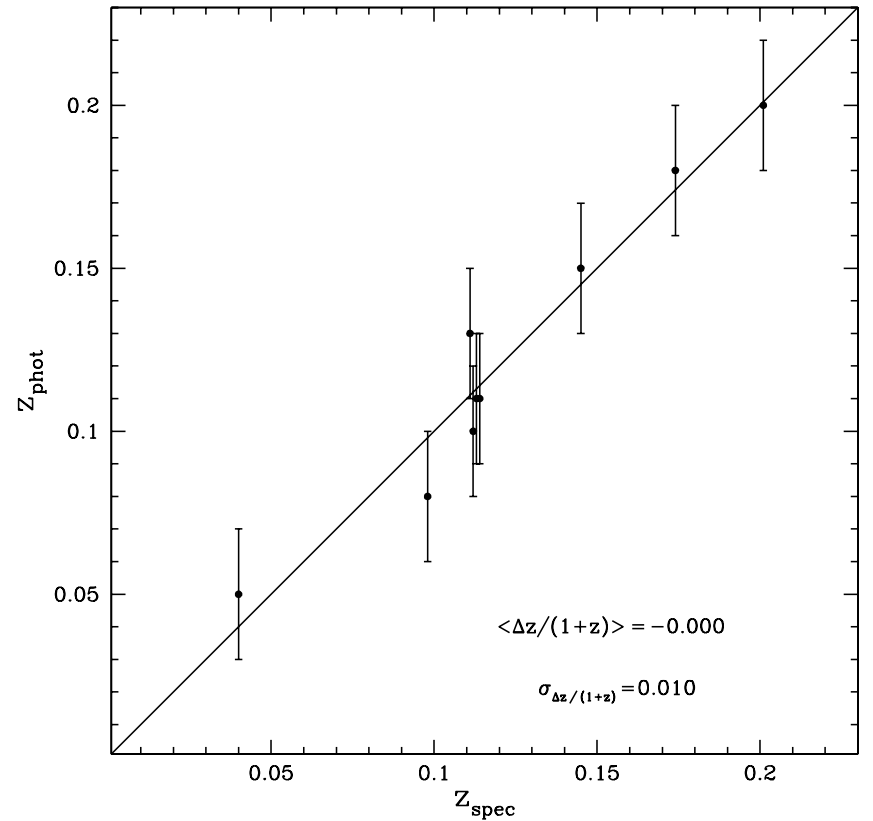

Figure 11. Comparison of spectroscopic and photometric redshifts for the available sample. This plot makes use of the results obtained with the S. F. Sánchez et al. (2008, in preparation) code described in the text. The statistics refer exclusively to the nine points represented in the plot.

et al. 2004). The value of the odds represents the fraction of the probability $p(z)$ concentrated around the maximum $z_{\mathrm{ph}}$. At fainter magnitudes, the photometric noise degrades the redshift information and often $p(z)$ is multimodal or presents a single peak of large width, making an unambiguous estimate of the redshift unfeasible. Therefore, by imposing cuts on the value of the odds, we can select galaxy subsamples for which the redshift estimates are reliable and accurate; in fact, it can be shown that the redshift accuracy depends on the severity of the threshold cut.

Our simulations predict that the galaxies with odds $>0.99$ and odds $>0.95$ will have photometric redshift errors of $\delta \leqslant 0.015(1+z)$ and $\delta \leqslant 0.03(1+z)$, respectively. The total number of galaxies that can be measured to a given accuracy level is given, as a function of the magnitude, in Figure 14. In Figure 15 the completeness level, as a function, is shown for different accuracy levels.

\section{COMPARISON WITH OTHER SURVEYS}

Several large, deep photometric, and spectroscopic surveys have been completed in recent years or are currently being conducted. Comparisons between different surveys, each of them designed for specific purposes and with different strategies, is not straightforward. To put the ALHAMBRA survey in the context of other similar efforts we have plotted in Figure 16 their positions in an area-depth plane. ${ }^{17}$ Only photometric surveys with five or more filters, i.e., those that can provide general redshift information, and an area covering $\geqslant 0.5 \mathrm{deg}^{2}$ (with the exception of CADIS) were included. As we can see in this plot, the ALHAMBRA survey occupies a position in between the first generation of wide field photometric surveys-among which COMBO-17 has clearly played the leading role up to now-and the ongoing projects that try to

\footnotetext{
17 Note that the magnitude limits correspond in general to different spectral bands. In the particular case of IRAS/PSCz, a typical $B$ magnitude has been taken as indicative of the population, selected in the $60 \mu \mathrm{m}$ band.
}

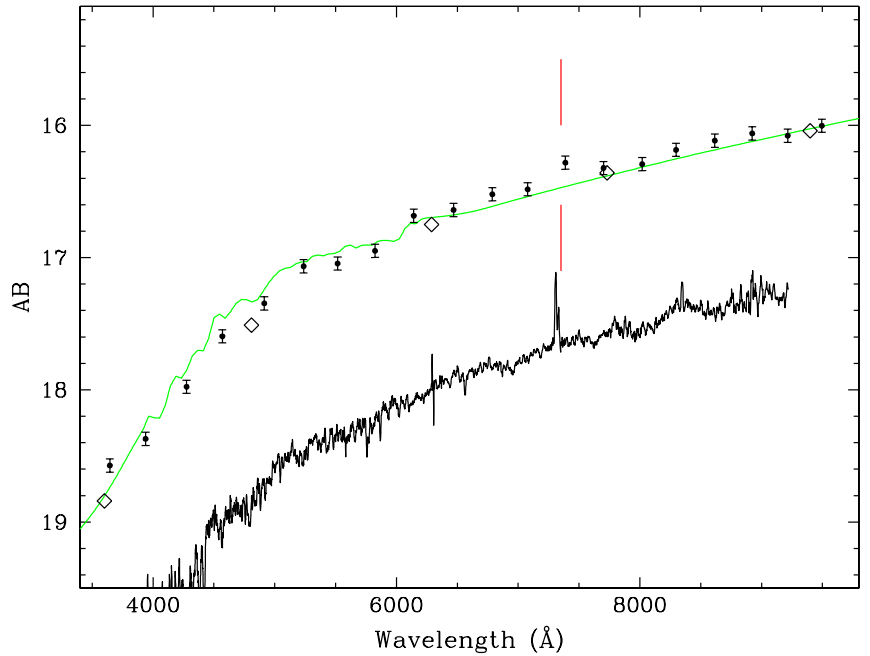

Figure 12. The ALHAMBRA spectrum (points with error bars), best-fit model (smooth line), and SDSS photometry (diamonds) and spectrum (lower, noisy line) for one of the galaxies with spectroscopic redshift in the ALHAMBRA-8 field. The vertical ticks mark the expected position of the $\mathrm{H} \alpha+\mathrm{N}$ II complex at the best-fit redshift. Note the apparent excess in the ALHAMBRA photometry at the expected wavelength, even though the total equivalent width measured for the complex in the SDSS spectrum is only $15 \AA$.

(A color version of this figure is available in the online journal.)

map much larger areas with a comparable depth such as the Panoramic Survey and Rapid Response System (Pan-Starrs), the Dark Energy Survey (DES), the Physics of the Accelerated Universe (PAU) survey, and the Large Synoptic Survey Telescope (LSST). COMBO-17 covers an area slightly larger than $1 \mathrm{deg}^{2}$ in five fields observed with the Wide-Field Imager at the MPG/ESO $2.2 \mathrm{~m}$ telescope in La Silla (Chile). The large number of bands (five broadband filters $U B V R I$ and 12 medium-band filters from $3500 \AA$ to $9300 \AA$ ) has provided accurate measurements of photometric redshifts (Wolf et al. 2001) to undertake ambitious scientific projects, two of the last scientific achievements being the measurement of galaxy clustering at moderate redshifts $(\langle z\rangle=0.6)$ (Phleps et al. 2006) and the analysis of 3D weak lensing (Kitching et al. 2007).

As we have already indicated, the ALHAMBRA survey has been designed to provide a deeper photometric survey in the northern hemisphere making use of 20 optical medium-band filters designed and optimized for the accurate determination of photometric redshifts (Benítez et al. 2008), covering four times the area covered by COMBO-17, and including from its design the standard NIR filters $J, H$, and $K$. We are using in ALHAMBRA the $3.5 \mathrm{~m}$ telescope at Calar Alto, and, therefore, the gain in aperture and the use of infrared filters will make the ALHAMBRA survey deeper than COMBO-17. It is worth saying that the COMBO-17 team has also planned an NIR extension of its survey that will allow it to reach $z \sim 2$ within its covered area (Meisenheimer et al. 2005).

The ALHAMBRA survey compares favorably with other deep, large photometric surveys, even though it uses mediumband filters. It is only second in limit magnitude to a few broadband photometric surveys. Indeed, comparing the ALHAMBRA survey (not to mention spectroscopic surveys) with broadband surveys in those terms is unfair, since the resulting accuracy in redshift and SED determination precision is significantly different. Thus, whereas typical redshift accuracies from those broadband surveys is $\sim 0.1$ in $\Delta z /(1+z)$ at best, we expect to reach 0.015 with ALHAMBRA for several hundred thousand galaxies. 


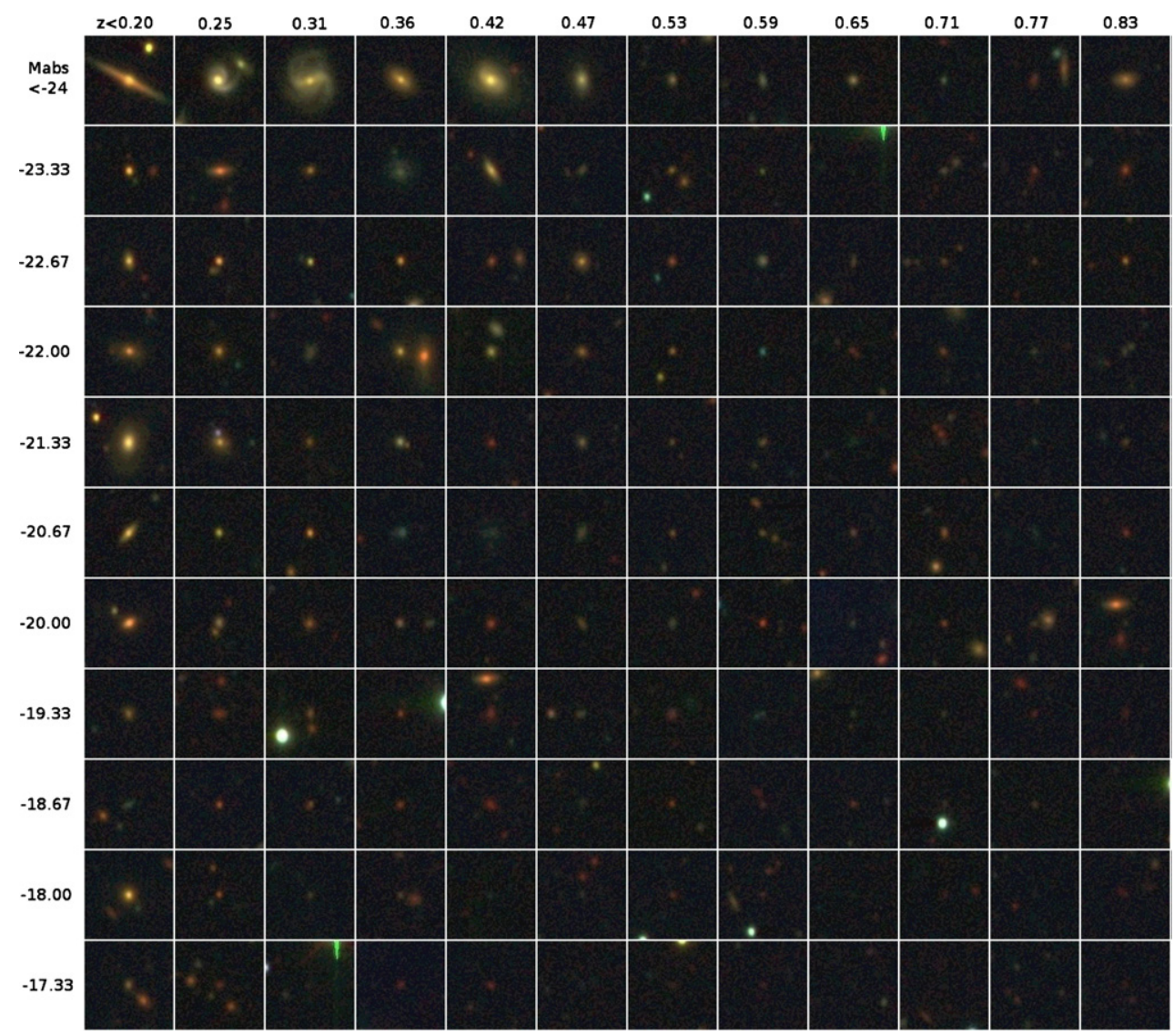

Figure 13. Postage stamp images of a set of galaxies extracted from the catalog of the field presented in this work. Each postage stamp has been generated using the $3960 \AA$ (blue), $6750 \AA$ (green), and $K_{s}$-band (red) frames to create a three-color image. The galaxies are ordered by increasing redshift in the $X$-axis and decreasing $7370 \AA$ absolute magnitude along the $Y$-axis, with each plotted galaxy being the most luminous of its corresponding redshift-luminosity box. The luminosity ranges from $M_{7370}<-24$ to $M_{7370} \approx-16.67$, and the redshift ranges from $z<0.20$ to $z=0.90$. Redshift and magnitude values for each column and line are given in the figure.

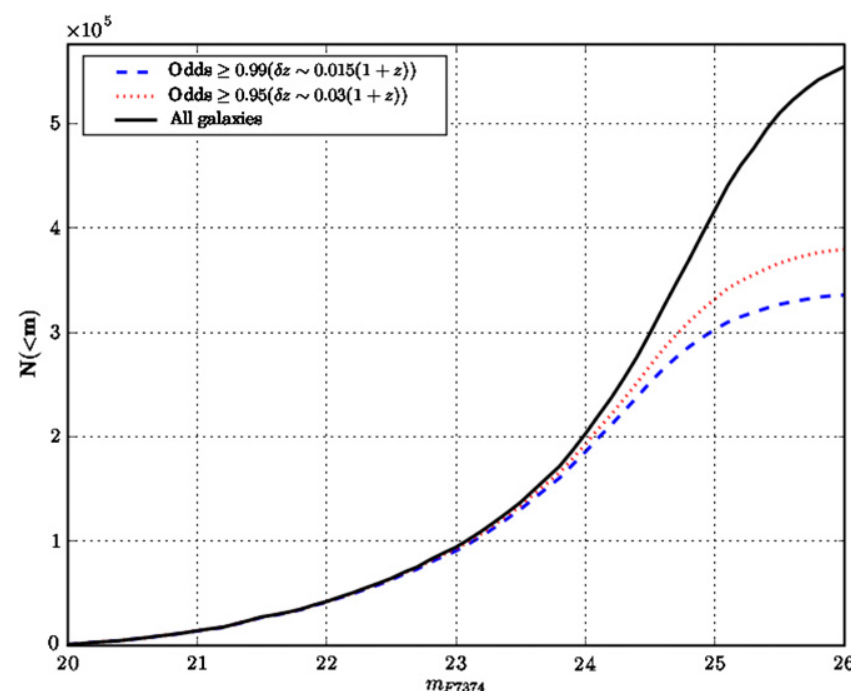

Figure 14. The total number of galaxies we expect to detect in the ALHAMBRA survey (thick continuous), where we use the $F 7374$ band as a proxy for the $I$ band. The dashed and dotted lines give the total number of galaxies with different odds thresholds (see the text). These results are based on our first complete $15^{\prime} \times 15^{\prime}$ arc $\min ^{2}$ pointing.

A recent effort quantitatively similar in some degree to ALHAMBRA is the one presented by Mobasher et al. (2007), who have measured photometric redshifts in the Cosmic Evolution Survey (COSMOS) covering an area of

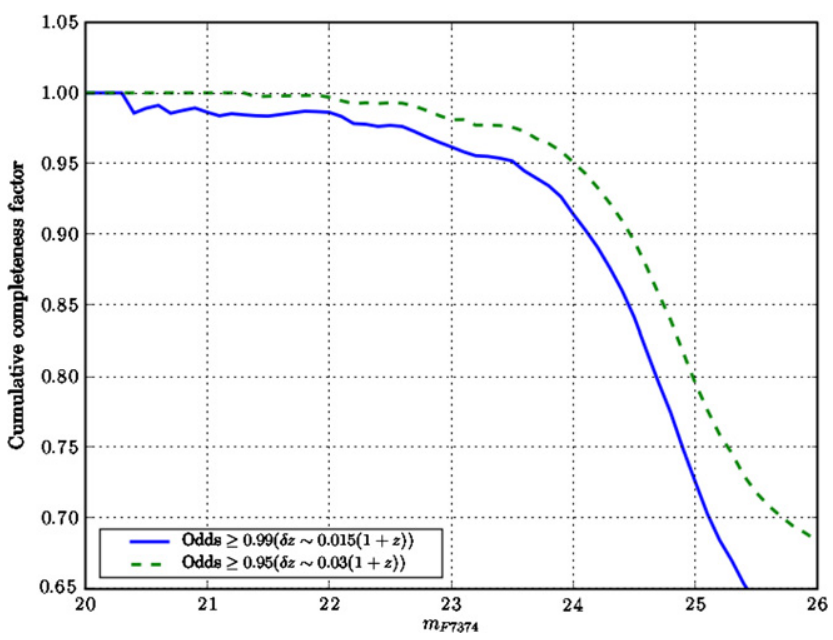

Figure 15. The expected completeness level for the ALHAMBRA survey as a function of magnitude; here we use the $F 7374$ band as a proxy for the $I$ band. The dashed and dotted lines correspond to different odds thresholds (see text). These results are based on our first complete $15^{\prime} \times 15^{\prime} \operatorname{arcmin}^{2}$ pointing.

$1.4^{\circ} \times 1.4^{\circ}$ containing 367,000 galaxies down to $i \sim 25$ using 16 filters and providing accurate redshifts for faint galaxies up to $z \sim 1.2$. The reliability of their measurement has been tested by comparing it with the spectroscopy subsample zCOSMOS, which contains 868 normal galaxies with $z<1.2$ (Lilly et al. 2007). 


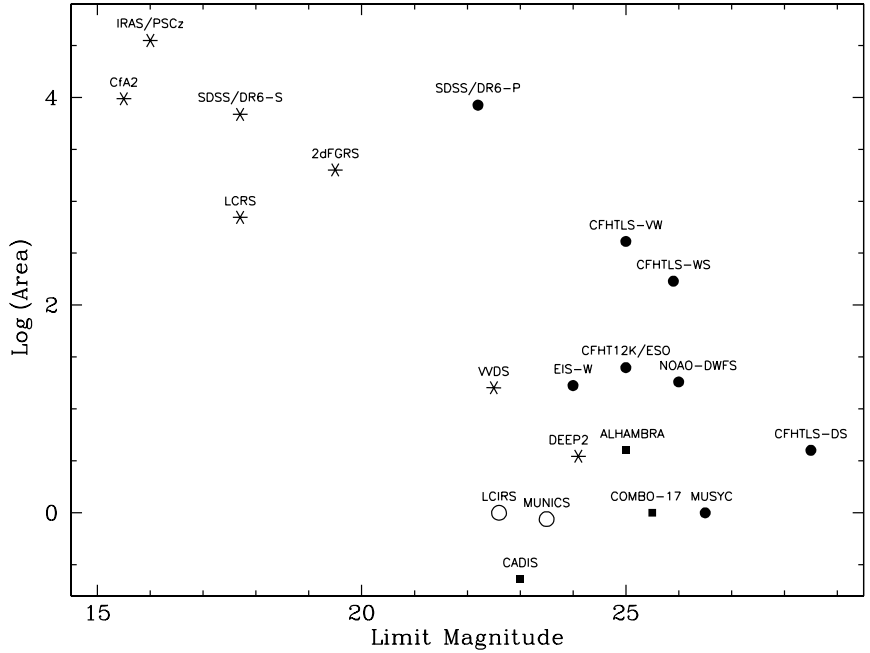

Figure 16. The position of different surveys in the area-depth plane. Only photometric surveys with a minimum of five filters and covering at least $0.5 \mathrm{deg}^{2}$ (with the exception of CADIS) are shown. The circles correspond to broadband photometric surveys, the stars to spectroscopic surveys, and the squares to CADIS, COMBO-17, and ALHAMBRA. The empty circles correspond to NIR imaging surveys.

Spectroscopic surveys do of course achieve the highest redshift and SED precision, but they cannot go as deep as photometric surveys, and do not always have a complete spectral coverage, resulting in complex selection functions. Besides, the detection limit is not homogeneous along the spectrum, resulting in a lack of completeness that can prove very difficult to control. In some cases (for example, when there is only one line in the spectrum), the photometric data can be used to reduce the ambiguity present in the spectroscopic measurements (Lilly et al. 2007). Given that our survey, in spite of its photometric nature, is closer in spirit to spectroscopic surveys, we discuss in the following paragraphs how it compares with them.

Indeed, the spectroscopic surveys can provide much more detailed information about individual objects than any mediumband photometric survey. However, for all those purposes where that detailed information is not needed, a survey like ALHAMBRA will prove advantageous due to the homogeneity of the detection in the different filters and the ability to produce accurate results even near the detection limit. Thus, as we noted before, we expect to be $60 \%$ complete down to $I=24.7 \mathrm{mag}$ with accurate $z$-determination, with a median redshift of 0.74 . In Figure 17, we have plotted the expected global performance of different spectroscopic surveys in terms of surveyed volume and number of objects with good SED and redshift determinations. The ALHAMBRA survey appears close to the SDSS in terms of the number of detected objects, and close to the deepest surveys in terms of its median redshift.

Another important advantage for the ALHAMBRA survey is the spectral range covered. It is, in fact, the only survey covering the whole optical domain, from $3500 \AA$ to $9700 \AA$, plus the three standard NIR bands. This implies that we are sensitive, within the detection limits, to any kind of object at any redshift. This not only avoids the presence of selection functions, but, more importantly, will straightforwardly allow the comparative analysis of data at different redshift values.

Table 3 shows the relevant data for the spectroscopic surveys plotted in Figure 17 (the number of objects in the ALHAMBRA survey is given for $\Delta z /(1+z) \leqslant 0.03$ and, in parenthesis, $\leqslant 0.015$ ). For surveys with a similar (even if smaller) spectral

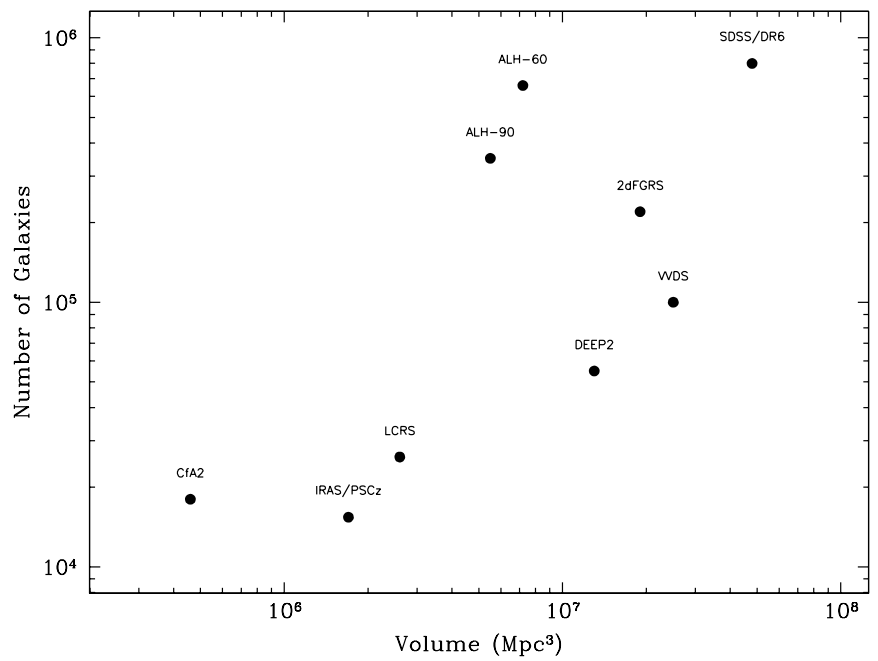

Figure 17. Number of detected objects with accurate redshift vs. the surveyed volume for spectroscopic surveys covering at least $0.5 \mathrm{deg}^{2}$, and the ALHAMBRA survey. We have considered a median redshift of 0.02 for CfA2 and IRAS/PSCz, 0.08 for LCRS, 0.1 for SDSS/DR6 and 2dF, 0.7 for VVDS, 1.0 for DEEP2, and 0.63 and 0.74 for ALHAMBRA-90 (complete at the $90 \%$ level) and ALHAMBRA-60 (complete at the $60 \%$ level) respectively. The plotted ALHAMBRA data are for $\Delta z /(1+z) \leqslant 0.03$.

coverage, such as SDSS or 2dFGRS, the ALHAMBRA survey will be 7.8 and $6.7 \mathrm{mag}$ deeper, respectively, which will allow, in particular, for a detailed analysis of the distant/faint universe. At the other extreme, surveys such as VVDS or DEEP2 that are not too far from the ALHAMBRA survey in terms of limit magnitude (even if they are shallower by 1.2 and $2.7 \mathrm{mag}$, respectively), have a significantly shorter spectral coverage than ALHAMBRA in the optical domain, and do not include NIR information.

Hickson \& Mulrooney (1998) presented the first results of the medium-band photometric survey proposed by Hickson et al. (1994). We note that the survey used more filters than ALHAMBRA, thus giving a finer spectral sampling, but their spectral coverage was shorter, from 4450 to $9480 \AA$. The observing conditions were such that the survey could reach only $\mathrm{m} \approx 20.4$, at the $50 \%$ completeness limit. Thus, even if similar in many aspects to our survey, a real comparison cannot be made given the bright detection limit they achieved.

In relation to other medium-band photometric surveys, covering the whole spectral range, we have mentioned COMBO17 and CADIS - which has also has made use of one of the Calar Alto telescopes - that comprise a mixed set of broad- and narrow-band filters. The complete spectral coverage is assured only by the broadband filters. Their specificity relies on the use of narrow filters located at some given fixed positions. The total area that they cover ( 1 and $0.2 \mathrm{deg}^{2}$, respectively), the photometric depth, and the lack of NIR information, are also important differences from the ALHAMBRA survey.

Yet another project making use of photometric redshifts and covering very large areas of the sky, but with a depth less than $z \sim 0.7$, is MegaZ-LRG (Collister et al. 2007), containing about a million SDSS luminous red galaxies in a region of $5915 \mathrm{deg}^{2}$, with a limiting magnitude $i<20$. This catalog, with a surveyed volume of about $2.5 \mathrm{~h}^{-3} \mathrm{Gpc}^{3}$, has been recently used to measure cosmological parameters from the large-scale structure in a competitive way to with the shallow wide-angle spectroscopic surveys (Blake et al. 2007). We must remark that an $M_{\star}$ galaxy at redshift $z \sim 1.4$ will be included in ALHAMBRA, since its 
Table 3

Main Characteristics of Wide-Field $\left(\geqslant 0.5 \mathrm{deg}^{2}\right)$ Spectroscopic Surveys

\begin{tabular}{lcccc}
\hline \hline Survey & Area & Spectral Range $(\AA)$ & $z$ (median) & $N_{\text {objects }}$ \\
\hline CfA/SRSS & 18000 & $4300-6900$ & 0.02 & $1.8 \times 10^{4}$ \\
SDSS/DR6 & 6860 & $3800-9200$ & 0.1 & $7.9 \times 10^{5}$ \\
LCRS & 700 & $3350-6750$ & 0.1 & $2.6 \times 10^{4}$ \\
2dFGRS & 2000 & $3700-8000$ & 0.11 & $2.2 \times 10^{5}$ \\
VVDS & 16 & $5500-9500$ & 0.7 & $1.0 \times 10^{5}$ \\
DEEP2 & 3.5 & $6500-9100$ & 1.0 & $5.5 \times 10^{3}$ \\
ALHAMBRA-60 & 4 & $3500-9700(+J H K)$ & 0.74 & $6.6(3.0) \times 10^{5}$ \\
ALHAMBRA-90 & 4 & $3500-9700(+J H K)$ & 0.63 & $3.5(1.0) \times 10^{5}$
\end{tabular}

visual magnitude should be about 24 . At this redshift, the whole ALHAMBRA survey covering $4 \mathrm{deg}^{2}$ will produce a volume of about $3 \times 10^{7} \mathrm{~h}^{-3} \mathrm{Mpc}^{3}$.

Other ongoing projects are the ESO/VLT Survey Telescope Kilo-Degree Survey (ESO/VST KIDS) that will cover $1400 \mathrm{deg}^{2}$ making use of images in four broad bands. Its major goal will be the study of weak lensing, although it should also be a good sample to study baryonic acoustic oscillations (Peacock et al. 2006), and the Dark Energy Survey, that makes use of a new camera on the CTIO $4 \mathrm{~m}$ telescope. This survey will cover $5000 \mathrm{deg}^{2}$, but no NIR observations are planned. Farther into the future, the LSST plans to map about half of the sky in the standard broad bands ugrizy to obtain about $3 \times 10^{9}$ photometric redshifts. The first light of this project is scheduled for 2012, and as Peacock et al. (2006) have pointed out, the lack of NIR information may be an issue.

Different research projects devoted to the study of the evolution of galaxies across the Hubble time will get great advantage from the ALHAMBRA survey, when the complete catalog is released as publicly available. For example, this survey will increase the reliability of the present studies of the growth of the population of red galaxies since $z \sim 1$ (Ford et al. 2006) or the evolution with redshift of the color-density relation (Cucciati et al. 2006; Cooper et al. 2007). Another field where a deep and wide catalog such as ALHAMBRA will be very useful is the analysis of the assembly history of red galaxies (Brown et al. 2008) or field spheroidals (Treu et al. 2005). Similarly, the ALHAMBRA survey covers enough area and is deep enough to accurately trace the evolution of the luminosity function for different spectral types (Poli et al. 2003; Treu et al. 2005; Wolf et al. 2003; Zucca et al. 2006; Marchesini et al. 2007) and the growth of stellar mass with cosmic time (Borch et al. 2006).

All in all, we can say that even if ALHAMBRA is a photometric survey, it shares important aspects with the spectroscopic surveys, with which it compares well in many respects. Its advantages, depending on the proposed goals, are the complete spectral coverage, the homogeneity in the detection level along the spectrum, and the depth.

\section{FINAL CONSIDERATIONS}

The ALHAMBRA survey places itself halfway between relatively shallow, limited spectral coverage, wide-area spectroscopic surveys, and deep, large area, broadband photometric surveys. By trying to optimally combine the advantages of each kind of survey, we intend to observe a large area $\left(4 \mathrm{deg}^{2}\right)$ using a specially designed set of 20 medium bands, minimally overlapping filters covering the whole visible range from $3500 \AA$ to $9700 \AA$, plus the standard $J H K_{s}$ NIR filters. It will provide homogeneous data down to $A B \approx 25$ for all the filters from $3500 \AA$ to $8500 \AA$, with a magnitude limit of $A B=23.2$ at
$9550 \AA$. The filter characteristics were decided to allow us to detect even relatively faint emission features (observed EW $\geqslant$ $30 \AA$ ).

Together with the NIR information to $K_{s} \approx 20, H \approx 21$, $J \approx 22$ (in the Vega system), this will allow the measurement of the redshift and SED for several hundred thousand objects. Indeed, the survey was designed with the use of photometric redshift techniques as the basic analysis tool. We have carried out detailed simulations based on available deep catalogs. We expect that the ALHAMBRA survey will be able to produce high-quality redshifts and accurate spectral types for more than 600,000 galaxies down to $I_{A B} \approx 24.7$, with redshift accuracy $\Delta z /(1+z) \approx 0.015$. The first data and preliminary results we present here confirm that these expectations are realistic.

With its volume surveyed, median redshift, and number of objects with accurate redshift and SED determination, the ALHAMBRA survey will provide a unique set of data for many different studies in different astrophysical and cosmological domains.

The main objective of our survey is the study of cosmological evolution, under the many facets it can offer. We will study the evolution of the large-scale structure, the number and content of clusters at different redshifts, the evolution of the populations of different cosmic objects, and the processes leading to galaxy formation, evolution, and differentiation. The unbiased nature of the survey will also allow for the study of many different kinds of objects, ranging from emission-line galaxies to the diverse types of AGNs and stars in our own Galaxy.

We acknowledge the decisive support given by the ALHAMBRA Extended Team to the project (see http://www.iaa. es/alhambra for the details regarding the project implementation and organization). We also wish to acknowledge the Calar Alto staff for their warm assistance in a fruitful start of the observations. We acknowledge the work of C. Cárdenas to measure the filter characteristics and of V. Peris for his work to produce the combined pictures out of the ALHAMBRA images. We thank our anonymous referee, whose comments helped to improve the clarity and quality of our manuscript. We acknowledge support from the Spanish Ministerio de Educación y Ciencia through grants AYA2002-12685-E, AYA2003-08729-C02-01, AYA2003-0128, AYA2004-08260C03-01, AYA2004-20014-E, AYA2004-02703, AYA200405395, AYA2005-06816, AYA2005-07789, AYA2006-14056, and from the Junta de Andalucía, TIC114, TIC101 and Proyecto de Excelencia FQM-1392. N.B., J.A.L.A., M.C., and A.F.S. acknowledge support from the MEC Ramón y Cajal Programme. N.B. acknowledges support from the EU IRG-017288. This work has made use of software designed at TERAPIX and the Canadian Astronomy Data Centre. 


\section{REFERENCES}

Adelman-McCarthy, J. K., et al. 2007, ApJS, 172, 634

Alcala, J. M., et al. 2002, Proc. SPIE, 4836, 406

Baum, W. A. 1962, IAU Symp., 15, 390

Becker, R. H., et al. 2001, AJ, 122, 2850

Beckwith, S. V. W., et al. 2006, AJ, 132, 1729

Bell, E. F., et al. 2004, ApJ, 608, 752

Benítez, N. 2000, ApJ, 536, 571

Benítez, N., et al. 2004, ApJS, 150, 1

Benítez, N., et al. 2008, A\&A, submitted

Bertin, E., \& Arnouts, S. 1996, A\&AS, 117, 393

Blake, C. A., Collister, A. A., Bridle, S. L., \& Lahav, O. 2007, MNRAS, 374,1527

Borch, A., et al. 2006, A\&A, 453, 869

Bradley, L. D., et al. 2008, ApJ, 678, 647

Brown, M., et al. 2008, arXiv:0804.2293

Coe, D., et al. 2006, AJ, 132, 926

Coleman, G. D., Wu, C.-C., \& Weedman, D. W. 1980, ApJS, 43, 393

Colless, M., et al. 2001, MNRAS, 328, 1039

Collister, A., et al. 2007, MNRAS, 375, 68

Connolly, A. J., Csabai, I., Szalay, A. S., Koo, D. C., Kron, R. G., \& Munn, J. A. 1995, AJ, 110, 2655

Connolly, A. J., Szalay, A. S., Dickinson, M., Subbarao, M. U., \& Brunner, R. J. 1997, ApJL, 486, 11

Cooper, M. C., et al. 2007, MNRAS, 376, 1445

Cucciati, O., et al. 2006, A\&A, 458, 39

Davis, M., et al. 2003, SPIE, 4834, 161

Ferguson, H. C., Dickinson, M., \& Williams, R. 2000, ARA\&A, 38, 667

Fernández-Soto, A., Lanzetta, K. M., Chen, H.-W., Pascarelle, S. M., \& Yahata, N. 2001, ApJS, 135, 1, 41

Fernández-Soto, A., Lanzetta, K. M., \& Yahil, A. 1999, ApJ, 513, 34

Ford, H., et al. 2006, IAU Symp., 235, in press

Fukugita, M., Ichikawa, T., Gunn, J. E., Doi, M., Shimasaku, K., \& Schneider, D. P. 1996, AJ, 111, 1748

Gregg, M. D., et al. 2004, BAAS, 36, 1496 http://lifshitz.ucdavis.edu/mgregg/ gregg/ngsl/ngsl.html

Hickson, P., Gibson, B. K., \& Callaghan, K. A. S. 1994, MNRAS, 267, 911
Hickson, P., \& Mulrooney, M. K. 1998, ApJS, 115, 35

Hildebrandt, H., Wolf, C., \& Benítez, N. 2008, A\&A, 480, 703

Ilbert, O., et al. 2006, A\&A, 457, 841

Kashikawa, N., et al. 2006, ApJ, 648, 7

Kawai, N., et al. 2006, Nature, 440, 184

Kitching, T. D., et al. 2007, MNRAS, 376, 771

Koo, D. C. 1986, ASSL, 122, 419

Lanzetta, K. M., Yahil, A., \& Fernández-Soto, A. 1996, Nature, 381, 751

Le Fèvre, O., et al. 2005, A\&A, 439, 845

Lilly, S. J., et al. 2007, ApJS, 172, 70

Loh, E. D., \& Spillar, E. J. 1986, ApJ, 303, 154

Marchesini, D., et al. 2007, ApJ, 656, 42

Márquez, I., \& Moles, M. 1996, A\&ASS, 119, 1

Massey, P., \& Gronwall, C. 1990, ApJ, 358, 344

Meisenheimer, K., et al. 1998, in ASP Conf. Series 146, The Young Universe: Galaxy Formation and Evolution at Intermediate and High Redshift, ed.

S. D’Odorico, A. Fontana, \& E. Giallongo (San Francisco, CA: ASP), 134

Meisenheimer, K., et al. 2005, MPI technical report

Mobasher, B., et al. 2007, ApJS, 172, 117

Moles, M., et al. 2005, arXiv astro-ph/0504545

Monet, D. G., et al. 2003, AJ, 125, 984

Oke, J. B. 1990, AJ, 99, 1621

Oke, J. B., \& Gunn, J. E. 1983, ApJ, 266, 713

Peacock, J., et al. 2006, Report by the ESA-ESO on Fundamental Cosmology (arXiv:astro-ph/0610906)

Phleps, S., et al. 2006, A\&A, 457, 145

Poli, F., et al. 2003, ApJL, 593, 1

Schlegel, D. J., Finkbeiner, D. P., \& Davis, M. 1998, ApJ, 500, 525

Smith, J. A., et al. 2002, AJ, 123, 2121

Stanford, S. A., Eisenhardt, P. R. M., \& Dickinson, M. 1995, ApJ, 450, 512

Stone, R. P. S. 1996, ApJS, 107, 423

Treu, T., et al. 2005, ApJ, 633, 174

Valdes, F. G. 2002, Automated Data Analysis in Astronomy, 309

Wolf, C., Maisenheimer, K., \& Röser, H.-J. 2001, A\&A, 365, 660

Wolf, C., et al. 2001, A\&A, 365, 861

Wolf, C., et al. 2003, A\&A, 401, 73

Wolf, C., et al. 2004, A\&A, 421, 913

York, D. G., et al. 2000, AJ, 120, 1579

Zucca, E., et al. 2006, A\&A, 455, 879 